\title{
Bryozoans from the lower Silurian (Telychian) Hanchiatien Formation from southern Chongqing, South China
}

\author{
Andrej Ernst, ${ }^{1}{ }^{\oplus}$ Qi-Jian Li, ${ }^{2,3,4}$ Min Zhang, ${ }^{5}$ and Axel Munnecke ${ }^{4}$ \\ ${ }^{1}$ Institut für Geologie, Universität Hamburg, Bundesstrasse 55, D-20146 Hamburg, Germany <Andrej.Ernst@ uni-hamburg.de> \\ ${ }^{2}$ State Key Laboratory of Palaeobiology and Stratigraphy, Nanjing Institute of Geology and Paleontology, East Beijing Road 39, Nanjing \\ 210008, China \\ ${ }^{3}$ Center for Excellence in Life and Paleoenvironment, Chinese Academy of Sciences, East Beijing Road 39, Nanjing 210008, China \\ ${ }^{4}$ GeoZentrum Nordbayern, Friedrich-Alexander University Erlangen-Nuremberg, D-91054 Erlangen, Germany <qijianli@ hotmail.com> \\ <axel.munnecke@fau.de> \\ ${ }^{5}$ School of Earth Sciences, China University of Geosciences, 388 Lumo Road, Wuhan, Hubei 430074, China <mzhangcug@163.com>
}

\begin{abstract}
Eight bryozoan species are described from the Hanchiatien Formation (lower Silurian, Telychian) of southern Chongqing, South China. Four species are new: the trepostomes Asperopora sinensis n. sp., Trematopora jiebeiensis n. sp., and Trematopora tenuis n. sp., and the fenestrate Moorephylloporina parvula n. sp. One species, the cystoporate Hennigopora sp. indet., is described in open nomenclature. Moorephylloporina parvula n. sp. is eurytopic, occurring in all types of facies within the bioherms. Erect Moorephylloporina Bassler, 1952, Trematopora Hall, 1852, and Leioclema Ulrich, 1882 formed pioneering communities on weakly cemented substrata, whereas encrusting Fistulipora M'Coy, 1849, Hennigopora Bassler, 1952, and Asperopora Owen, 1969 occurred on hardgrounds and formed densely compact framestones. Robust branched Trematopora and Leioclema tend to occur out of the reef core (framework) where they could have formed reef-flank thickets in more agitated conditions. The generic composition of the studied fauna correlates with other localities in South China, and they show general paleobiogeographic relations to Siberia and Indiana, USA.
\end{abstract}

UUID http://zoobank.org/3326dd2f-7c9e-43bc-9dab-84047b274f89

\section{Introduction}

Lower Silurian (Llandovery) marine sediments are distributed along a NNE-SSW trend on the South China plate, which was largely occupied by the Yangtze region in the northwestern sector. During the Llandovery, the Yangtze region has been interpreted to represent a tropical or subtropical 'epeiric platform' in western Gondwana (e.g., Rong and Cocks, 2014). In the earliest Silurian (Rhuddanian stage), black shales with flysch are dominant sediments in the Yangtze region. Due to the rapid rise of sea level, late Aeronian limestone facies became widespread in the epeiric sea of the Yangtze region, however, the early Telychian is dominated by terrigenous sediments with minor calcareous interbeds due to the expansion of the Yangtze Uplift (Rong et al., 2003, 2012; Deng et al., 2012). The early Telychian rocks are represented by the Hanchiatien (or Majiaochong) Formation, usually over a kilometer in thickness, spanning from northern Guizhou to southern Sichuan provinces and the southern Chongqing District. Li et al. (2018) provided an in-depth study of the metersized, bryozoan-bearing bioherms, described preliminarily earlier in Chinese by Li et al. (2012). The bryozoan fauna in those bioherms have not yet been given a systematic treatment. Previous studies of the early Telychian bryozoan fauna of the Yangtze region are scarce (Hu, 1982; Xia and Qi, 1989), thus, a detailed description of the Hanchiatien bryozoans can provide valuable insights into the faunal composition in the region.
Telychian bryozoans from South China were previously described in several papers. Hu (1982) identified six bryozoan species from the Cuijiagou Formation (lower Telychian) of Guangyuan, Sichuan: Fistulipora guangyuanensis Hu, 1982, Fistuliramus eximius $\mathrm{Hu}$, 1982, Hennigopora multilamellosa $\mathrm{Hu}$, 1982, Hennigopora sp. indet., Trematopora sinensis $\mathrm{Hu}$ 1982, and Trematopora sp. indet. From the upper Telychian Ningqiang Formation of Ningqiang (Shanxi), he identified 14 species (Hu, 1982, 1990): Calloporella silurica Hu, 1982, Cyclotrypa solidoscens $\mathrm{Hu}, 1990$, Cyphotrypa undulata $\mathrm{Hu}$, 1990, Fistulipora ningqiangensis Hu, 1990, Fistuliramus transversus $\mathrm{Hu}, 1982$, Hennigopora petaliformis $\mathrm{Hu}, 1982$, Hallopora aggregata $\mathrm{Hu}, 1982$, Homotrypa ningqiangensis $\mathrm{Hu}, 1990$, Leioclema speciosum $\mathrm{Hu}, 1982$, Leioclema sp. indet., Monotrypa shaanxiensis $\mathrm{Hu}, 1990$, Orbignyella curvata $\mathrm{Hu}, 1982$, O. globata Yang, 1951, and O. mui Yang, 1951.

Xia and Qi (1989) described four species from the lower Telychian Chenxiacun Formation of Hanshan (Anhui): Hallopora elegantula (Hall, 1852), Hallopora hanshanensis Xia and Qi, 1989, Hallopora raritabulata Xia and Qi, 1989, and Trematopora reflua Xia and Qi, 1989.

\section{Geological settings and depositional environment}

The Hanchiatien Shale was originally defined by Ting (1930) and was subsequently treated as the Hanchiatien Group (e.g., 


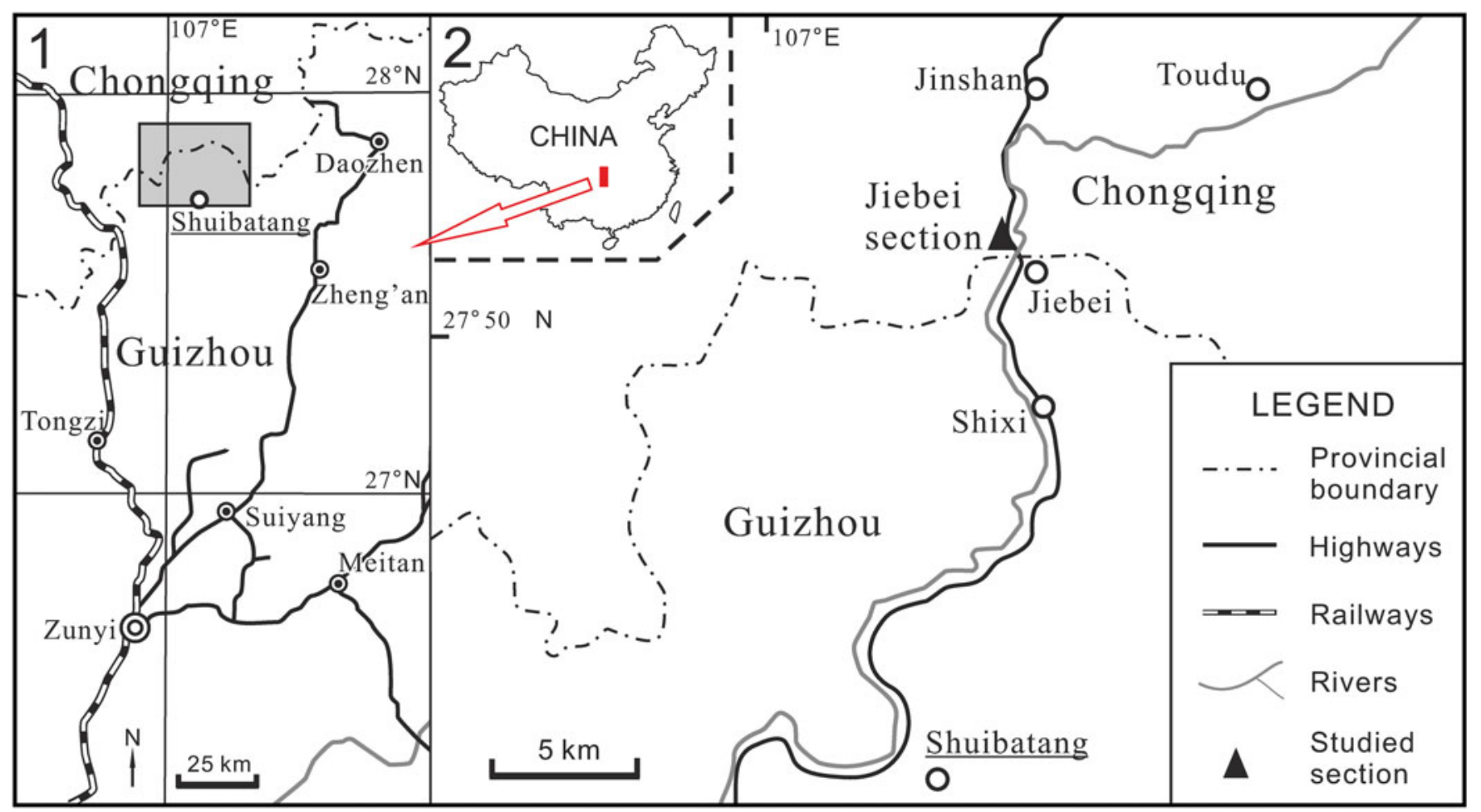

Figure 1. Location of the studied section between Chongqing and Guizhou provinces, South China: (1) region between Chongqing and Guizhou provinces; (2) detail of the shaded-box area in (1), showing the study section.

Yin, 1949; Mu, 1962). Since 1978, it has been formally named the Hanchiatien Formation (e.g., Southwest Institute of Geologic Sciences, 1978; Rong et al., 1990), characterized by brownish to greenish shales. The shales are usually disconformably underlain by the upper Aeronian Shihniulan Formation and disconformably capped by the lower Permian Liangshan or Tongkuangxi formations (Zhan and Jin, 2007). In most cases, there is a unit (normally $<10 \mathrm{~m}$ thick) of purplish red shales known as the Lower Red Beds at the base of the Hanchiatien Formation. Stratigraphically, the Lower Red Beds are a wellknown marker unit in the Yangtze region (Rong et al., 2012).

Although the Hanchiatien Formation is poorly fossiliferous, diagnostic chitinozoans and graptolites were reported at the top of the formation in the Northeast Guizhou: Ancyrochitina brevicollis Geng, 1986 and Streptograptus plumosus Baily, 1871 suggest the formation should be correlated to early Telychian (Chen, 1986; Geng et al., 1997). Some carbonate interbeds (or marls) occur in the Hanchiatien Formation in a few localities (e.g., Li et al., 2012). Macrofossils (e.g., brachiopods, trilobites, echinoderms, bryozoans, and corals) are abundant in some of the carbonate interbeds. To date, no systematic study has been done on the bryozoans from this formation. Here, we provide the first taxonomic description of the bryozoans from the lower Hanchiatien Formation at the border of Guizhou Province.

The Hanchiatien Formation is thought to have been deposited in a subtidal setting, mainly based on the distributional pattern of the brachiopods (Rong et al., 2003). However, different facies of the Hanchiatien Formation display a fluctuation of marine environments ( $\mathrm{Li}$ et al., 2018). The paleogeographical setting of the Hanchiatien Formation indicates a seaward ramp oriented northward. Depositional environments could have varied from place to place in the Yangtze epeiric sea.

The Jiebei section is located at the boundary between Chongqing and Guizhou provinces (Fig. 1). The stratigraphic details were given by Li et al. (2018) who described the lower Hanchiatien Formation as brownish to greenish shales with minor silstones, which cap the Shihniulan Formation disconformably. In this section, there are two bioherms $85-120 \mathrm{~m}$ above the base of the Hanchiatien Formation. At the bottom of the second bioherm, there is a layer ( $\sim 1.7 \mathrm{~m}$ thick) of cross-bedded, oolitic grainstone with ripples (Fig. 2). Lacking any photic-related fossils, the bryozoan bioherms demonstrate an unusual heterozoan marine community, mainly produced by internal waves $(\mathrm{Li}$ et al., 2018). Our interpretation is that the lower Hanchiatien Formation in Jiebei represents mid-outer ramp settings.

\section{Material and methods}

All bryozoans were collected from the bioherms (Figs. 2, 3) in June 2016 by QJL. Thin sections were made in the laboratory of the GeoZentrum Nordbayern, Friedrich-Alexander University Erlangen-Nuremberg, Germany. Bryozoans were studied in thin sections using a binocular microscope under transmitted light. In total, 42 thin sections were prepared from rock samples. The spacing of structures is measured as the distance between their centers. Statistics were summarized using arithmetic means, sample standard deviations, coefficients of variation, and minimum and maximum values.

Based on the genus-level occurrence databases of Silurian bryozoans (Hu, 1982, 1990; Xia and Qi, 1989; Anstey et al., 2003), pair-group cluster analysis (Euclidean similarity index) 


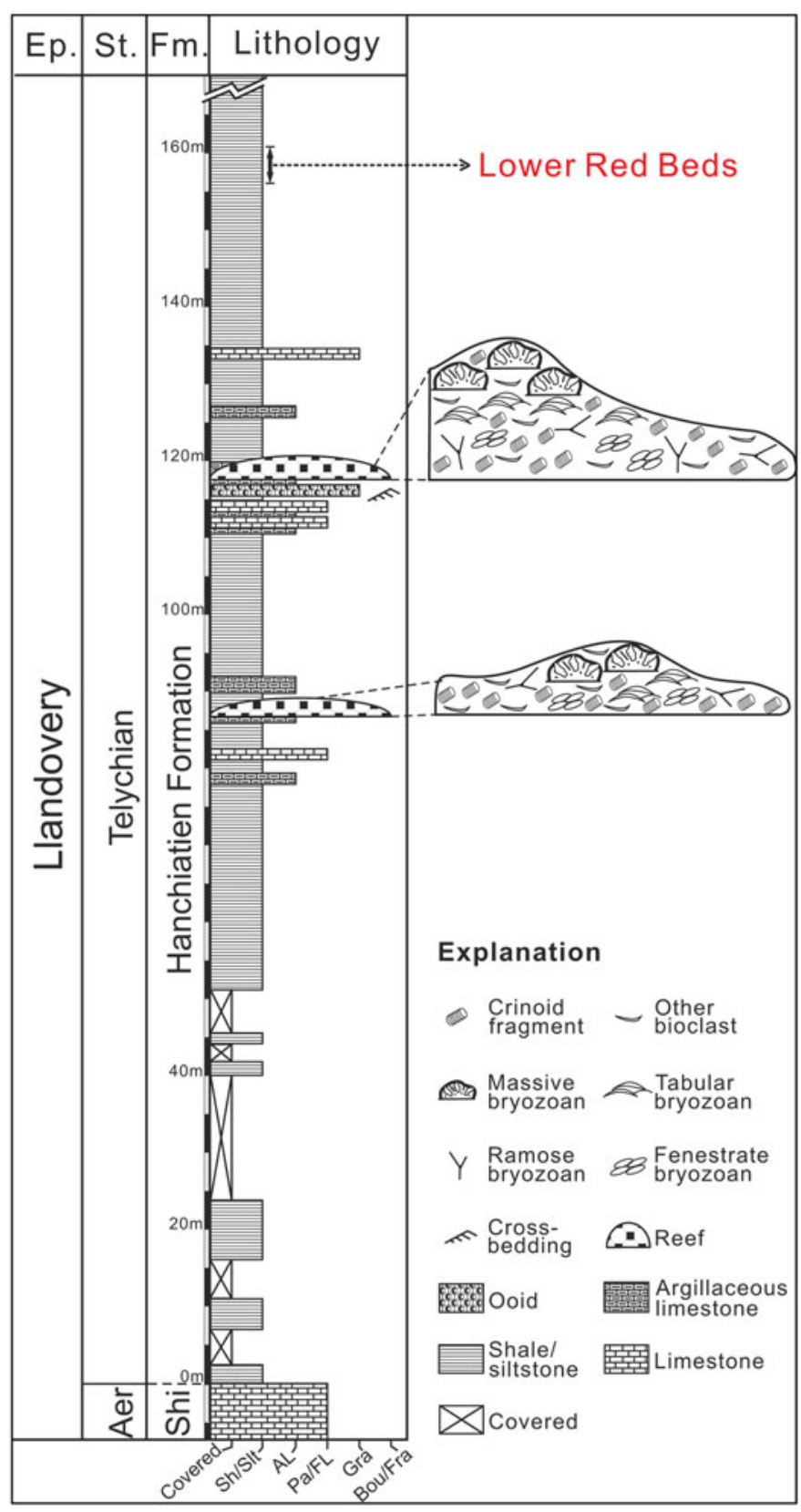

Figure 2. Lithologic column of the Jiebei section (from Li et al., 2018). Stratigraphic positions of reefs are indicated. Aer $=$ Aeronian; $\mathrm{AL}=$ argillaceous limestone; Bou/Fra = boundstone/framestone; Ep. = epoch; Fm. = formation; Gra = grainstone $; \mathrm{Pa} / \mathrm{FL}=$ packstone/floatstone; $\mathrm{Sh} / \mathrm{Slt}=$ shale/siltstone $; \mathrm{Shi}=$ Shihniulan Formation; St. $=$ stage.

and detrended correspondence analysis were implemented with the PAST statistical package (version 2.16; Hammer et al., 2001) to assess the paleobiogeographical relationship of the Telychian bryozoan fauna from the South China Block. Both cluster analysis and detrended correspondence analysis make no assumptions about the data structure, so they were suitable for our large-scale paleobiogeographical studies (Shi, 1993). To ensure the robustness of our results, taxa that were potentially poorly known were removed from the published presence-absence dataset (see Appendix).
Repositories and institutional abbreviations.-The newly studied material is deposited at the Naturmuseum Senckenberg (SMF), Frankfurt am Main, Germany. Other cited repositories are: NIGP $=$ Nanjing Institute of Geology and Paleontology, China.

\section{Systematic paleontology}

Phylum Bryozoa Ehrenberg, 1831

Class Stenolaemata Borg, 1926

Superorder Palaeostomata Ma, Buttler, and Taylor, 2014

Order Cystoporata Astrova, 1964

Suborder Fistuliporina Astrova, 1964

Family Fistuliporidae Ulrich, 1882

Genus Fistulipora M`Coy, 1849

Type species._Fistulipora minor M`Coy, 1849; Carboniferous, England.

Fistulipora guangyuanensis $\mathrm{Hu}, 1982$ Figure 4.1-4.5; Table 1

1982 Fistulipora guangyuanensis Hu, p. 296, pl. 3, figs. 11, 12.

Holotype.-NIGP 63771; Cuijiagou Formation, Telychian, Llandovery, lower Silurian; Sichuan Province, China.

Occurrence.-Jiebei village, Chongqing, China; lower part of the Hanchiatien Formation, lower Telychian, Llandovery, lower Silurian.

Materials._SMF 60500-60538.

Remarks._Fistulipora guangyuanensis differs from Fistulipora ningqiangensis from the lower Silurian of Ningqiang, China in its smaller apertures (aperture width $0.12-0.18 \mathrm{~mm}$ vs. $0.17-0.25 \mathrm{~mm}$ [Hu, 1990], respectively) and larger lunaria. Fistulipora guangyuanensis differs from Fistulipora ternavensis Astrova, 1965 from the lower-middle Silurian of Russia in its smaller autozooecial apertures (aperture width $0.12-0.18 \mathrm{~mm}$ vs. 0.18-0.23 mm [Astrova, 1965], respectively), larger lunaria, and less abundant vesicles (7-9 per autozooecial aperture vs. 8-10 vesicles [Astrova, 1965], respectively).

Family Xenotrypidae Utgaard, 1983 Hennigopora Bassler, 1952

Type species.—Callopora florida Hall, 1852; Niagaran Group, Rochester Shale, Sheinwoodian, Wenlock, Silurian; Lockport, New York, USA.

\section{Hennigopora multilamellosa $\mathrm{Hu}, 1982$} Figure 4.6-4.10; Table 2

1982 Hennigopora multilamellosa Hu, p. 295, pl. 2, figs. 4, 5. Holotype.-NIGP 63765; Cuijiagou Formation, Telychian, Llandovery, lower Silurian; Sichuan Province, China. 

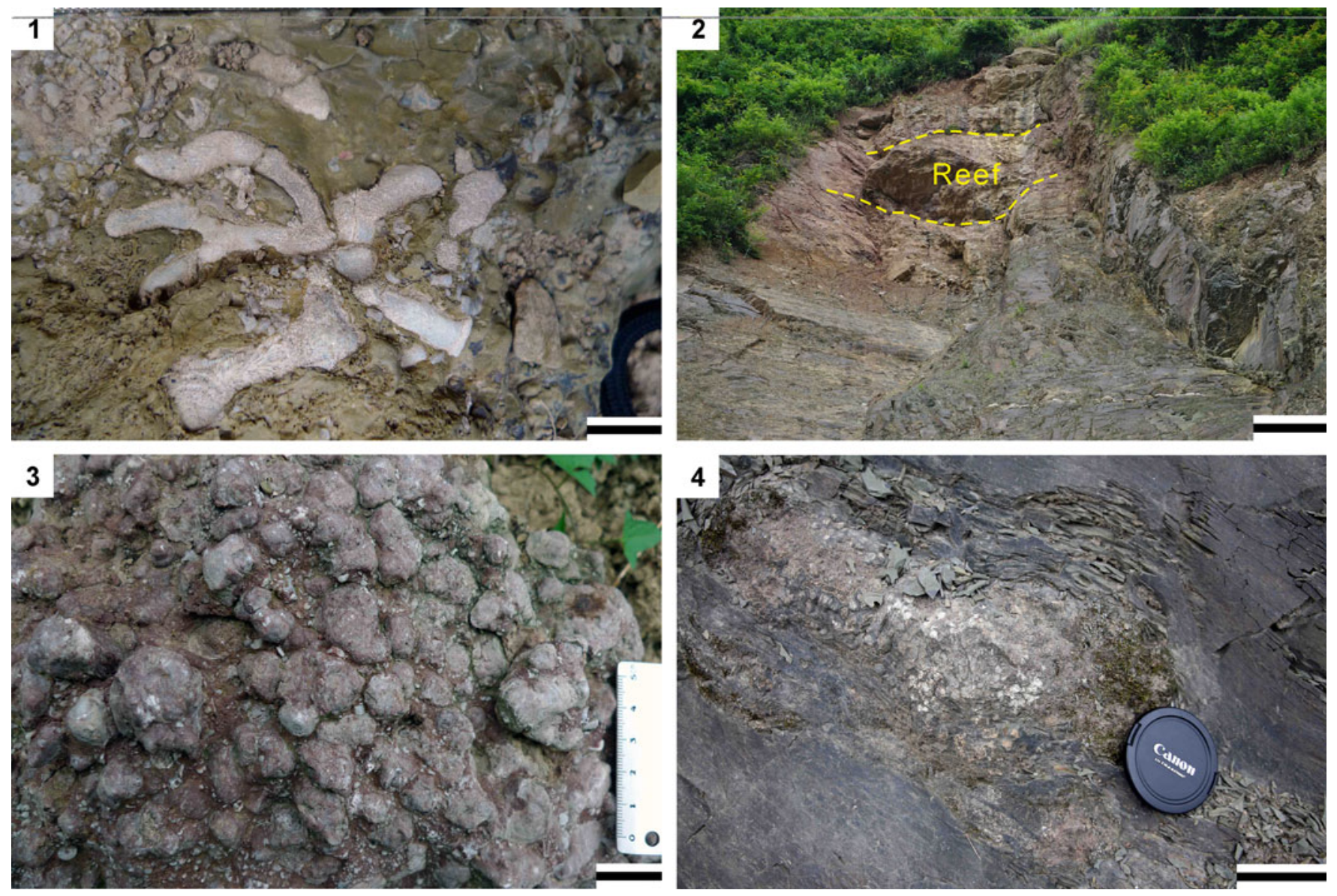

Figure 3. Field aspects of the studied section: (1) the flank facies of the second reef horizon, showing the branched bryozoan Trematopora; (2) the core facies of the second reef horizon; (3) plan view of the framestone, showing bulbous and knobby colonies of the first reef horizon; (4) the flank facies of the first reef horizon. Scale bars $=2 \mathrm{~m} \mathrm{(2)} ; 8 \mathrm{~cm} \mathrm{(4);2} \mathrm{cm} \mathrm{(3);1} \mathrm{cm}(\mathbf{1})$.

Occurrence.-Jiebei village, Chongqing, China; lower part of the Hanchiatien Formation, lower Telychian, Llandovery, lower Silurian.

\section{Materials.-SMF 60539-60576.}

Remarks.-Hennigopora multilamellosa differs from Hennigopora florida (Hall, 1852) from the middle Silurian of New York, USA in its smaller autozooecial apertures (aperture width $0.17-0.28 \mathrm{~mm}$ vs. $0.28-0.35 \mathrm{~mm}$ [Bassler, 1906], respectively). Hennigopora multilamellosa differs from Hennigopora hunanensis Yang and Xia, 1974 from the lower Silurian of China in having smaller autozooecial apertures (aperture width $0.17-0.28 \mathrm{~mm}$ vs. $0.20-0.30 \mathrm{~mm}$ [Yang and Xia, 1974], respectively) and in its less abundant vesicles (6-11 per aperture vs. 8-13, respectively). Hennigopora multilamellosa differs from Hennigopora apta Perry and Hattin, 1960 from the lower Silurian of Indiana, USA in the presence of 4-7 acanthostyles per autozooecial aperture instead of 3-5 (Ernst et al., 2019) in the latter species. Hennigopora multilamellosa differs from Hennigopora petaliformis from the Ningqiang Formation (upper Telychian) of Shanxi in its encrusting instead of branched erect colony and in the presence of 4-7 acanthostyles per autozooecial aperture instead of 2-4 (Hu, 1982) in the latter species.
Hennigopora sp. indet.

Figure 4.11-4.15; Table 3

Occurrence.--Jiebei village, Chongqing, China; lower part of the Hanchiatien Formation, lower Telychian, Llandovery, lower Silurian.

Description.-Encrusting colony, $0.40-1.25 \mathrm{~mm}$ thick. Autozooecia originating from thin epitheca, bending in the early exozone to the colony surface, with roundedpolygonal, often petaloid, apertures due to indenting acanthostyles. Basal diaphragms rare, straight, thin. Vesicles generally large, separating autozooecia in one or two rows, 8 -10 surrounding each autozooecial aperture, with rounded roofs, polygonal in tangential section. Acanthostyles abundant, relatively large, often deeply indenting autozooecial chambers, six or seven surrounding each autozooecial aperture. Autozooecial walls displaying obscure granular microstructure, $0.005-0.010 \mathrm{~mm}$ thick. Maculae not observed.

Materials._Single specimen, SMF 60577.

Remarks.-Hennigopora sp. indet. differs from Hennigopora multilamellosa in its smaller autozooecial apertures (mean aperture width $0.15 \mathrm{~mm}$ vs. $0.22 \mathrm{~mm}$ [Table 2], respectively) 

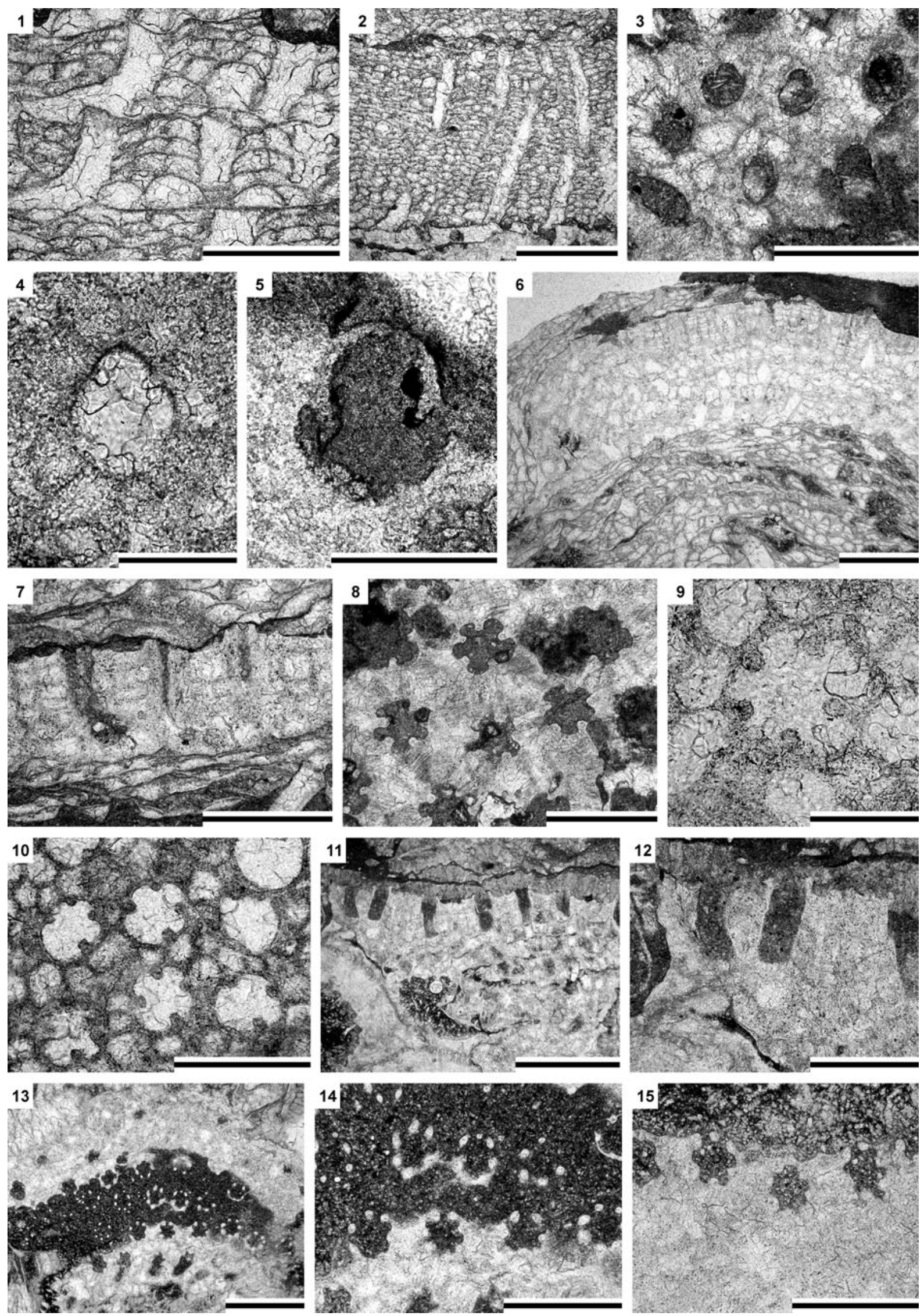
Figure 4. (1-5) Fistulipora guangyuanensis Hu, 1982: (1) longitudinal section of a multilayered encrusting colony, SMF 60526; (2) longitudinal section of an encrusting colony, SMF 60526; $(\mathbf{3}, \mathbf{4})$ tangential section showing autozooecial apertures and vesicles, SMF $60525 ;(\mathbf{5})$ tangential section showing autozooecial aperture with lunarium, SMF 60524; (6-10) Hennigopora multilamellosa Hu, 1982: (6) longitudinal section, SMF 60570; (7) longitudinal section showing autozooecia and vesicles, SMF 60552; (8-10) tangential sections showing autozooecial apertures, acanthostyles, and vesicles: (8) SMF 60544; (9) SMF 60542; (10) SMF 60554; (11-15) Hennigopora sp. indet., SMF 60577: $(\mathbf{1 1}, \mathbf{1 2})$ longitudinal section of an encrusting colony; $(13-15)$ tangential sections showing autozooecial apertures, acanthostyles, and vesicles. Scale bars $=1 \mathrm{~mm}(\mathbf{2}, \mathbf{6}, \mathbf{1 1}, \mathbf{1 3}) ; 0.5 \mathrm{~mm}(\mathbf{1}, \mathbf{3}, \mathbf{7}, \mathbf{8}, \mathbf{1 0}, \mathbf{1 2}, \mathbf{1 4}, \mathbf{1 5}) ; 0.2 \mathrm{~mm}(\mathbf{4}, \mathbf{5}, \mathbf{9})$.

Table 1. Descriptive statistics of Fistulipora guangyuanensis Hu, 1982. $\mathrm{CV}=$ coefficient of variation; $\mathrm{Max}=$ maximum value; $\mathrm{Min}=$ minimum value; $\mathrm{N}=$ number of measurements; $\mathrm{SD}=$ sample standard deviation; $\mathrm{X}=$ mean.

\begin{tabular}{lcccrcc}
\hline & $\mathrm{N}$ & $\mathrm{X}$ & $\mathrm{SD}$ & $\mathrm{CV}$ & $\mathrm{MIN}$ & $\mathrm{MAX}$ \\
\hline Aperture width (mm) & 30 & 0.15 & 0.014 & 9.65 & 0.12 & 0.18 \\
Aperture spacing (mm) & 30 & 0.34 & 0.039 & 11.62 & 0.23 & 0.40 \\
Vesicle diameter (mm) & 30 & 0.08 & 0.020 & 25.63 & 0.04 & 0.12 \\
Vesicles per aperture & 20 & 8.25 & 0.639 & 7.74 & 7.0 & 9.0 \\
Lunaria width (mm) & 30 & 0.10 & 0.013 & 13.62 & 0.07 & 0.12 \\
Lunaria length (mm) & 30 & 0.07 & 0.014 & 20.20 & 0.05 & 0.10 \\
Vesicle spacing (mm) & 30 & 0.07 & 0.014 & 20.20 & 0.05 & 0.10 \\
\hline
\end{tabular}

and more abundant acanthostyles (mean 6.6 acanthostyles per autozooecial aperture vs. 5 [Table 2], respectively). Hennigopora sp. indet. differs from Hennigopora apta from the lower Silurian of Indiana, USA in the presence of six or seven acanthostyles per autozooecial aperture instead of 3-5 (Ernst et al., 2019) in the latter species.

Order Trepostomata Ulrich, 1882

Suborder Halloporina Astrova, 1965

Family Heterotrypidae Ulrich, 1890

Genus Leioclema Ulrich, 1882

[= Lioclema Ulrich, 1882]

Type species.-Callopora punctata Hall, 1858; lower Carboniferous, Iowa, USA.

\section{Leioclema speciosum $\mathrm{Hu}, 1982$}

Figure 5.1-5.6; Table 4

1982 Leioclema speciosum Hu, p. 295, pl. 2, figs. 9-13. 1990 Leioclema speciosum; Hu, p. 29, pl. 2, figs. 4-6.

Holotype.-NIGP 63767; Cuijiagou Formation, Telychian, Llandovery, lower Silurian; Sichuan Province, China.

Occurrence.-Jiebei village, Chongqing, China; lower part of the Hanchiatien Formation, lower Telychian, Llandovery, lower Silurian.

Materials._SMF 60578-60598.

Table 2. Descriptive statistics of Hennigopora multilamellosa Hu, 1982. Abbreviations as for Table 1.

\begin{tabular}{lcccccc}
\hline & $\mathrm{N}$ & $\mathrm{X}$ & $\mathrm{SD}$ & $\mathrm{CV}$ & $\mathrm{MIN}$ & MAX \\
\hline Aperture width (mm) & 55 & 0.22 & 0.029 & 13.19 & 0.17 & 0.28 \\
Aperture spacing (mm) & 55 & 0.39 & 0.046 & 11.85 & 0.28 & 0.50 \\
Vesicle diameter (mm) & 55 & 0.10 & 0.024 & 23.05 & 0.06 & 0.16 \\
Acanthostyle diameter (mm) & 55 & 0.05 & 0.010 & 19.88 & 0.03 & 0.08 \\
Acanthostyles per aperture & 55 & 5.0 & 0.680 & 13.65 & 4.0 & 7.0 \\
Vesicles per aperture & 55 & 9.3 & 1.171 & 12.56 & 6.0 & 11.0 \\
Vesicle spacing (mm) & 45 & 0.08 & 0.028 & 35.35 & 0.04 & 0.15 \\
\hline
\end{tabular}

Table 3. Descriptive statistics of Hennigopora sp. indet. Abbreviations as for Table 1.

\begin{tabular}{lcccccl}
\hline & $\mathrm{N}$ & $\mathrm{X}$ & $\mathrm{SD}$ & $\mathrm{CV}$ & $\mathrm{MIN}$ & $\mathrm{MAX}$ \\
\hline Aperture width (mm) & 20 & 0.15 & 0.010 & 6.79 & 0.14 & 0.17 \\
Aperture spacing (mm) & 20 & 0.32 & 0.039 & 12.20 & 0.25 & 0.37 \\
Vesicle diameter (mm) & 20 & 0.08 & 0.017 & 19.76 & 0.06 & 0.12 \\
Acanthostyle diameter (mm) & 20 & 0.031 & 0.004 & 14.04 & 0.025 & 0.038 \\
Acanthostyles per aperture & 17 & 6.6 & 0.507 & 7.70 & 6.0 & 7.0 \\
Vesicle spacing (mm) & 10 & 0.08 & 0.023 & 27.61 & 0.05 & 0.11 \\
\hline
\end{tabular}

Remarks._Leioclema speciosum is similar to Leioclema tuvaensis Astrova, 1959 from the Wenlock of Tuva (Russia), but differs in having smaller colonies and less abundant and smaller acanthostyles (acanthostyle diameter 0.030-0.045 mm vs. 0.01-0.03 $\mathrm{mm}$ [Astrova, 1959], respectively). Leioclema speciosum differs from Leioclema densiporum Owen, 1965 from the Llandovery of England in having larger autozooecial apertures (aperture width $0.10-0.20 \mathrm{~mm}$ vs. $0.10-0.12 \mathrm{~mm}$ [Owen, 1965], respectively).

\section{Genus Asperopora Owen, 1969}

Type species.-Callopora aspera Hall, 1852; Silurian (Wenlock), New York, USA.

\section{Asperopora sinensis new species}

Figure 5.7-5.11; Table 5

Type specimens.-Holotype, SMF 60599; paratypes, SMF 60600-60635.

Diagnosis.-Thin encrusting colonies; endozones short; autozooecial apertures rounded-polygonal; basal diaphragms few to common in exozone; one or two acanthostyles surrounding each autozooecial aperture; 6-10 mesozooecia surrounding each autozooecial aperture; maculae absent.

Occurrence.-Jiebei village, Chongqing, China; lower part of the Hanchiatien Formation, lower Telychian, Llandovery, lower Silurian.

Description.—Encrusting colonies, $0.4-1.0 \mathrm{~mm}$ thick. Autozooecia budding from a thin epitheca, growing a short distance parallel to the substratum, then bending sharply to the colony surface. Epitheca $0.003-0.005 \mathrm{~mm}$ thick. Autozooecial apertures rounded-polygonal. Basal diaphragms common in exozone, thin, slightly deflected orally. Acanthostyles common, one or two surrounding each autozooecial aperture, small, originating in the outer exozone, having distinct calcite cores and dark, laminated sheaths, indenting into autozooecial space. Mesozooecia abundant, 

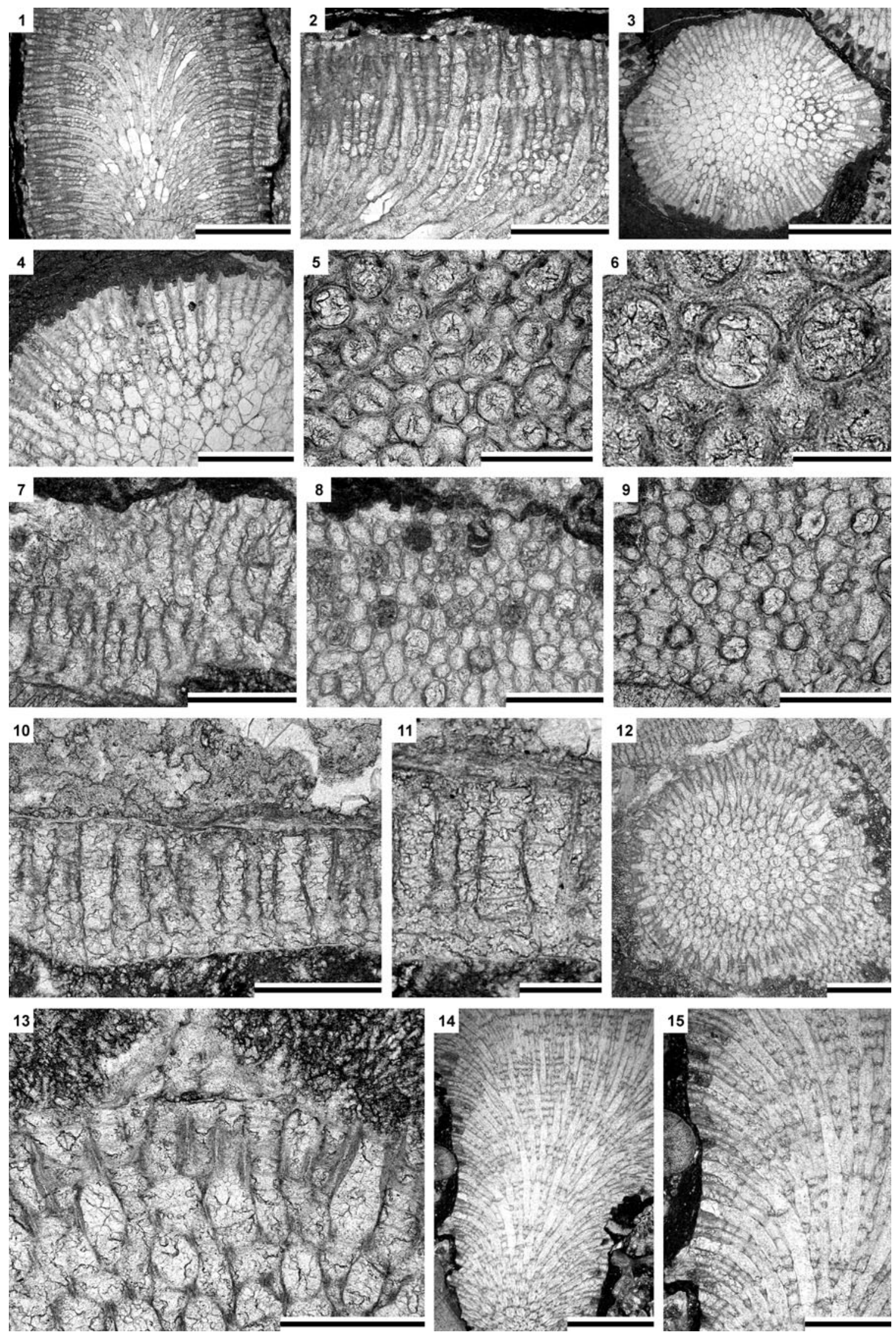
Figure 5. (1-6) Leioclema speciosum Hu, 1982: (1) longitudinal section of branched colony, SMF 60587; (2) longitudinal section of exozonal part of branched colony, SMF 60587; $(\mathbf{3 , 4})$ transverse section of branched colony, SMF 60586; $(\mathbf{5 , 6})$ tangential sections showing autozooecial apertures, acanthostyles, and mesozooecia, SMF 60587; (7-11) Asperopora sinensis n. sp.: (7) longitudinal section, holotype, SMF 60599; (8, 9) tangential sections showing autozooecial apertures, acanthostyles, and mesozooecia, holotype, SMF 60599; $(\mathbf{1 0}, \mathbf{1 1})$ longitudinal section showing autozooecia and mesozooecia, paratype, SMF 60606; (12-15) Trematopora jiebeiensis $\mathrm{n}$. sp.: $(\mathbf{1 2}, \mathbf{1 3})$ branch transverse section, paratype, SMF 60644; $(\mathbf{1 4}, \mathbf{1 5})$ branch longitudinal section, paratype, SMF 60643 . Scale bars $=2$ $\mathrm{mm}(\mathbf{1}, \mathbf{3}, \mathbf{1 4}) ; 1 \mathrm{~mm}(\mathbf{2}, \mathbf{4}, \mathbf{1 2}, \mathbf{1 5}), 0.5 \mathrm{~m}(\mathbf{5}, \mathbf{7}-\mathbf{1 0}, \mathbf{1 3}) ; 0.2 \mathrm{~mm}(\mathbf{6}, \mathbf{1 1})$.

6-10 surrounding each autozooecial aperture, roundedpolygonal in transverse section, originating at the base of exozone, slightly beaded. Autozooecial walls granular, 0.003-0.005 $\mathrm{mm}$ thick in endozone, laminated, merged, $0.02-0.03 \mathrm{~mm}$ thick in exozone. Maculae not observed.

Etymology.-The species is named after its occurrence in China.

Remarks.-Asperopora sinensis n. sp. differs from Asperopora bellum (Pushkin, 1976) from the middle Silurian of Belarus and Norway in its slightly smaller autozooecial apertures (mean aperture width $0.11 \mathrm{~mm}$ vs. $0.14 \mathrm{~mm}$ [Pushkin, 1976], respectively) and in its more abundant mesozooecia (mean 8.12 per aperture vs. 7.4 [Pushkin, 1976], respectively). Asperopora sinensis $\mathrm{n}$. sp. differs from Asperopora aspera (Hall, 1852) from the middle Silurian of North America and Europe in its less abundant and larger mesozooecia (mean 8.12 per aperture vs. 10.5 [Ernst et al., 2015], respectively), and in having one or two acanthostyles around each autozooecial aperture instead of 1-5 (Ernst et al., 2015) in the latter species.

\section{Family Trematoporidae Miller, 1889} Genus Trematopora Hall, 1852

Type species.-Trematopora tuberculosa Hall, 1852; lower Silurian (Niagaran), North America.

Trematopora jiebeiensis new species

Figures 5.12-5.15, 6.1-6.3; Table 6

Type specimens.-Holotype, SMF 60636; paratypes, SMF 60637-60660.

Diagnosis.-Branched colonies with distinct exozones; autozooecial apertures rounded to slightly angular; basal diaphragms rare; mesozooecia beaded, 5-8 surrounding each autozooecial aperture; acanthostyles moderately large,

Table 4. Descriptive statistics of Leioclema speciosum Hu, 1982. Abbreviations as for Table 1 .

\begin{tabular}{lcccccc}
\hline & $\mathrm{N}$ & $\mathrm{X}$ & $\mathrm{SD}$ & $\mathrm{CV}$ & $\mathrm{MIN}$ & $\mathrm{MAX}$ \\
\hline Branch width $(\mathrm{mm})$ & 10 & 3.16 & 0.743 & 23.56 & 2.40 & 4.50 \\
Exozone width (mm) & 10 & 0.75 & 0.154 & 20.56 & 0.45 & 0.90 \\
Endozone width (mm) & 10 & 1.66 & 0.647 & 38.98 & 0.78 & 2.78 \\
Aperture width (mm) & 70 & 0.13 & 0.022 & 16.80 & 0.10 & 0.20 \\
Aperture spacing (mm) & 70 & 0.22 & 0.033 & 15.26 & 0.16 & 0.30 \\
Mesozooecia width (mm) & 70 & 0.08 & 0.020 & 25.57 & 0.03 & 0.13 \\
Mesozooecia per aperture & 40 & 7.5 & 1.339 & 17.80 & 6.0 & 11.0 \\
Acanthostyle diameter (mm) & 20 & 0.036 & 0.005 & 14.54 & 0.030 & 0.045 \\
Acanthostyles per aperture & 20 & 3.0 & 0.918 & 30.59 & 2.0 & 5.0 \\
Mesozooecial diaphragm spacing & 70 & 0.09 & 0.020 & 21.73 & 0.05 & 0.15 \\
$\quad(m m)$ & & & & & & \\
Exozone wall thickness (mm) & 20 & 0.038 & 0.009 & 22.57 & 0.025 & 0.055 \\
\hline
\end{tabular}

2-6 surrounding each autozooecial aperture, originating in endozone; maculae absent.

Occurrence.-Jiebei village, Chongqing, China; lower part of the Hanchiatien Formation, lower Telychian, Llandovery, lower Silurian.

Description.-Ramose branched colonies; branch width 2.25-5.20 mm. Exozone distinct, 0.45-1.13 $\mathrm{mm}$ wide; endozone 1.35-3.26 mm wide. Secondary overgrowths common. Autozooecia long, polygonal in cross section in endozone, bending sharply in exozone, with rounded to slightly angular apertures. Basal diaphragms rare, thin, concentrated mainly in the transition between exo- and endozones. Mesozooecia abundant, originating at base of exozone, beaded in places of development of diaphragms, 5-8 surrounding each autozooecial aperture. Diaphragms in mesozooecia straight, abundant. Acanthostyles moderately large, prominent, having distinct hyaline cores, 2-6 surrounding each autozooecial aperture, originating in endozone. Autozooecial walls $0.003-0.005 \mathrm{~mm}$ thick, granular-prismatic in endozone, showing reversed V-shaped lamination, integrated with locally visible dark border between zooecia, 0.02-0.06 $\mathrm{mm}$ thick in exozone. Maculae absent.

Etymology.-The species is named after the type locality, the village of Jiebei in China.

Remarks.-Trematopora jiebeiensis n. sp. differs from Trematopora sinensis from the lower Silurian (Llandovery) of China in having smaller autozooecial apertures (aperture width 0.07-0.14 $\mathrm{mm}$ vs. $0.30-0.36 \mathrm{~mm}$ [Hu, 1982], respectively). Trematopora jiebeiensis n. sp. differs from Trematopora whitfieldi Ulrich, 1883 from the Silurian (Wenlock) of North America in its smaller autozooecial apertures (mean aperture width $0.11 \mathrm{~mm}$ vs. $0.14 \mathrm{~mm}$ [Ulrich, 1883], respectively).

\section{Trematopora tenuis new species} Figure 6.4-6.9; Table 7

Type specimens.-Holotype, SMF 60661; SMF 60662-60667.

Diagnosis.-Thin, branched colonies with distinct exozones; autozooecial apertures rounded to slightly angular; basal

Table 5. Descriptive statistics of Asperopora sinensis n. sp. Abbreviations as for Table 1.

\begin{tabular}{lcccccc}
\hline & $\mathrm{N}$ & $\mathrm{X}$ & $\mathrm{SD}$ & $\mathrm{CV}$ & $\mathrm{MIN}$ & MAX \\
\hline Aperture width $(\mathrm{mm})$ & 50 & 0.11 & 0.015 & 13.28 & 0.09 & 0.15 \\
Aperture spacing $(\mathrm{mm})$ & 50 & 0.25 & 0.037 & 14.84 & 0.17 & 0.34 \\
Mesozooecia width $(\mathrm{mm})$ & 50 & 0.07 & 0.017 & 22.37 & 0.03 & 0.11 \\
Acanthostyle diameter $(\mathrm{mm})$ & 43 & 0.03 & 0.007 & 21.37 & 0.02 & 0.045 \\
Mesozooecia per aperture & 50 & 8.12 & 1.003 & 12.35 & 6.0 & 10.0 \\
Mesozooecial diaphragm spacing (mm) & 50 & 0.06 & 0.021 & 34.35 & 0.02 & 0.10 \\
\hline
\end{tabular}



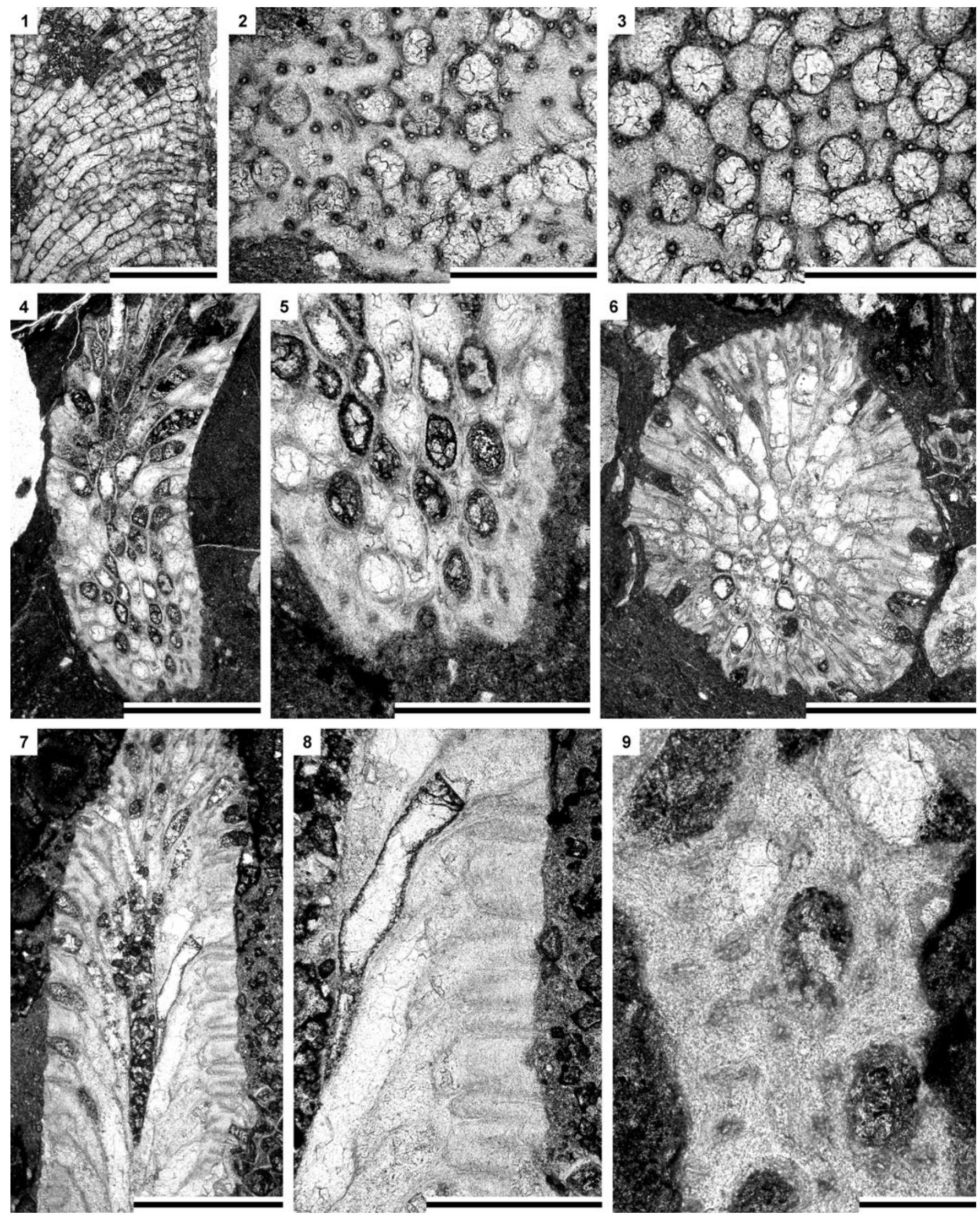

Figure 6. (1-3) Trematopora jiebeiensis n. sp.: (1) branch longitudinal section showing secondary overgrowth, autozooecia, mesozooecia, and acanthostyles, holotype, SMF 60636; (2,3) tangential sections showing autozooecial apertures, acanthostyles, and mesozooecia, holotype, SMF 60636; (4-9) Trematopora tenuis n. sp., holotype, SMF 60661: $(\mathbf{4 , 5})$ oblique section of a branch; $(\mathbf{6})$ branch transverse section; $(\mathbf{7 , 8})$ longitudinal section of a branch showing autozooecia and acanthostyles (9) tangential sections showing autozooecial apertures, acanthostyles, and mesozooecia. Scale bars = $1 \mathrm{~mm}(\mathbf{1}, \mathbf{4 ,} 6-\mathbf{6}) ; 0.5 \mathrm{~mm}(\mathbf{2}, \mathbf{3}, \mathbf{5}) ; 0.2 \mathrm{~mm}(\mathbf{9})$. 
Table 6. Descriptive statistics of Trematopora jiebeiensis $\mathrm{n}$. sp. Abbreviations as for Table 1.

\begin{tabular}{lcccccc}
\hline & $\mathrm{N}$ & $\mathrm{X}$ & $\mathrm{SD}$ & $\mathrm{CV}$ & $\mathrm{MIN}$ & $\mathrm{MAX}$ \\
\hline Branch width $(\mathrm{mm})$ & 10 & 3.63 & 1.043 & 28.77 & 2.25 & 5.20 \\
Exozone width $(\mathrm{mm})$ & 10 & 0.64 & 0.211 & 33.15 & 0.45 & 1.13 \\
Endozone width $(\mathrm{mm})$ & 10 & 2.35 & 0.705 & 30.00 & 1.35 & 3.26 \\
Aperture width $(\mathrm{mm})$ & 40 & 0.11 & 0.017 & 15.24 & 0.07 & 0.14 \\
Aperture spacing $(\mathrm{mm})$ & 40 & 0.19 & 0.023 & 11.76 & 0.15 & 0.25 \\
Acanthostyle diameter $(\mathrm{mm})$ & 40 & 0.04 & 0.008 & 20.29 & 0.03 & 0.06 \\
Mesozooecia width (mm) & 40 & 0.08 & 0.020 & 24.91 & 0.04 & 0.13 \\
Acanthostyles per aperture & 40 & 4.1 & 0.859 & 21.08 & 2.0 & 6.0 \\
Mesozooecia per aperture & 30 & 6.4 & 0.809 & 12.70 & 5.0 & 8.0 \\
Mesozooecial diaphragm spacing $(\mathrm{mm})$ & 40 & 0.08 & 0.027 & 32.27 & 0.04 & 0.16 \\
\hline
\end{tabular}

diaphragms rare; mesozooecia common, slightly beaded; acanthostyles moderately large, 6-8 surrounding each autozooecial aperture, originating in endozone; maculae absent.

Occurrence.-Jiebei village, Chongqing, China; lower part of the Hanchiatien Formation, lower Telychian, Llandovery, lower Silurian.

Description.-Ramose branched colonies; branch width $0.72-1.88 \mathrm{~mm}$. Exozone distinct, $0.20-0.38 \mathrm{~mm}$ wide; endozone $0.32-1.12 \mathrm{~mm}$ wide. Secondary overgrowths not observed. Autozooecia long, polygonal in cross section in endozone, bending sharply in exozone, with rounded to slightly angular apertures. Basal diaphragms rare, thin, concentrated mainly in the transition between exo- and endozones. Mesozooecia common, originating at base of exozone, slightly beaded in places of development of diaphragms. Diaphragms in mesozooecia straight, abundant. Acanthostyles moderately large, prominent, having distinct hyaline cores, 6-8 surrounding each autozooecial aperture, originating at the base of exozone. Autozooecial walls $0.003-0.005 \mathrm{~mm}$ thick, granular-prismatic in endozone, showing reversed $\mathrm{V}$-shaped lamination, integrated with locally visible, dark border between zooecia, 0.003-0.005 $\mathrm{mm}$ thick in exozone. Maculae absent.

Etymology.-The species is named because of its thin branches (Latin tenuis $=$ thin, narrow).

Remarks.-Trematopora tenuis $\mathrm{n}$. sp. is similar to Trematopora cristata Kopajevitch, 1984 from the Wenlock of Mongolia, but differs from the latter in having more abundant acanthostyles (6-8 per autozooecial aperture vs. 1-4 [Kopajevitch, 1984], respectively). Trematopora tenuis $\mathrm{n}$. sp. differs from Trematopora minima Ernst in Suttner and Ernst, 2007 from the Upper Ordovician of India in its less abundant mesozooecia and more abundant acanthostyles (6-8 per autozooecial aperture vs. four or five [Suttner and Ernst, 2007], respectively).

Order Fenestrata Elias and Condra, 1957

Suborder Fenestellina Astrova and Morozova, 1956

Family Fenestellidae King, 1849

Genus Moorephylloporina Bassler, 1952

Type species.-Moorephylloporina typica Bassler, 1952; Middle Ordovician, Black Riverian, Virginia, USA.
Table 7. Descriptive statistics of Trematopora tenuis $\mathrm{n}$. sp. Abbreviations as for Table 1.

\begin{tabular}{lrccrcc}
\hline & $\mathrm{N}$ & $\mathrm{X}$ & $\mathrm{SD}$ & $\mathrm{CV}$ & MIN & MAX \\
\hline Branch width (mm) & 4 & 1.28 & 0.479 & 37.56 & 0.72 & 1.88 \\
Exozone width $(\mathrm{mm})$ & 4 & 0.29 & 0.084 & 29.57 & 0.20 & 0.38 \\
Endozone width (mm) & 4 & 0.71 & 0.327 & 46.45 & 0.32 & 1.12 \\
Aperture width (mm) & 15 & 0.08 & 0.008 & 9.45 & 0.07 & 0.09 \\
Aperture spacing (mm) & 15 & 0.21 & 0.021 & 10.23 & 0.17 & 0.24 \\
Acanthostyle diameter (mm) & 15 & 0.04 & 0.006 & 16.83 & 0.03 & 0.05 \\
Mesozooecia width (mm) & 15 & 0.06 & 0.009 & 16.54 & 0.04 & 0.07 \\
\hline
\end{tabular}

Moorephylloporina parvula new species

Figure 7.1-7.8; Table 8

Type specimens.-Holotype, SMF 60668; paratypes SMF 60669-60710.

Diagnosis.-Reticulate colonies with straight branches; dissepiments short, wide; fenestrules small, oval; autozooecial apertures rounded, two or three spaced per fenestrule length; keel wide, low, with high and moderately large nodes; hemisepta lacking; diaphragms present; vesicular skeleton present.

Occurrence.-Jiebei village, Chongqing, China; lower part of the Hanchiatien Formation, lower Telychian, Llandovery, lower Silurian.

Description.-Reticulate colonies with straight, frequently bifurcating branches, joined by short, wide dissepiments. Autozooecia arranged in two alternating rows on branches, having circular apertures with moderately high peristomes, two or three spaced per length of a fenestrule. Peristomes containing 10-12 nodes. Peristomal nodes $0.015-0.020 \mathrm{~mm}$ in diameter. Fenestrules oval. Keels wide, low. Keel nodes high, with moderate diameter and spacing, rounded to oval in their cross sections. Microacanthostyles on the reverse colony surface abundant, regularly spaced in longitudinal rows, $0.010-0.015 \mathrm{~mm}$ in diameter.

Interior description.-Autozooecia long, rectangular in the mid-tangential section, with well developed vestibule; axial wall straight; aperture positioned at distal end of chamber. Hemisepta absent. Diaphragms present. Internal granular skeleton thin,

Table 8. Descriptive statistics of Moorephylloporina parvula $\mathrm{n}$. sp. Abbreviations as for Table 1.

\begin{tabular}{lcccrcc}
\hline & $\mathrm{N}$ & $\mathrm{X}$ & $\mathrm{SD}$ & $\mathrm{CV}$ & MIN & MAX \\
\hline Branch width (mm) & 20 & 0.24 & 0.028 & 11.73 & 0.19 & 0.29 \\
Branch thickness (mm) & 10 & 0.25 & 0.022 & 9.03 & 0.22 & 0.29 \\
Dissepiment width (mm) & 10 & 0.17 & 0.026 & 15.64 & 0.13 & 0.21 \\
Fenestrule width (mm) & 10 & 0.21 & 0.037 & 17.96 & 0.16 & 0.26 \\
Fenestrule length (mm) & 10 & 0.39 & 0.042 & 10.80 & 0.34 & 0.46 \\
Distance between branch centers (mm) & 10 & 0.44 & 0.057 & 13.11 & 0.34 & 0.56 \\
Distance between dissepiment centers & 10 & 0.58 & 0.051 & 8.90 & 0.52 & 0.65 \\
$\quad(\mathrm{~mm})$ & & & & & & \\
Aperture width (mm) & 20 & 0.08 & 0.006 & 7.68 & 0.07 & 0.09 \\
Aperture spacing along branch (mm) & 20 & 0.24 & 0.022 & 9.13 & 0.20 & 0.28 \\
Apertures per fenestrule & 10 & 2.5 & 0.527 & 21.08 & 2.0 & 3.0 \\
Maximal chamber width (mm) & 10 & 0.07 & 0.008 & 11.40 & 0.06 & 0.09 \\
Node width (mm) & 10 & 0.06 & 0.011 & 19.20 & 0.04 & 0.07 \\
Distance between node centers (mm) & 10 & 0.24 & 0.040 & 16.35 & 0.19 & 0.30 \\
\hline
\end{tabular}



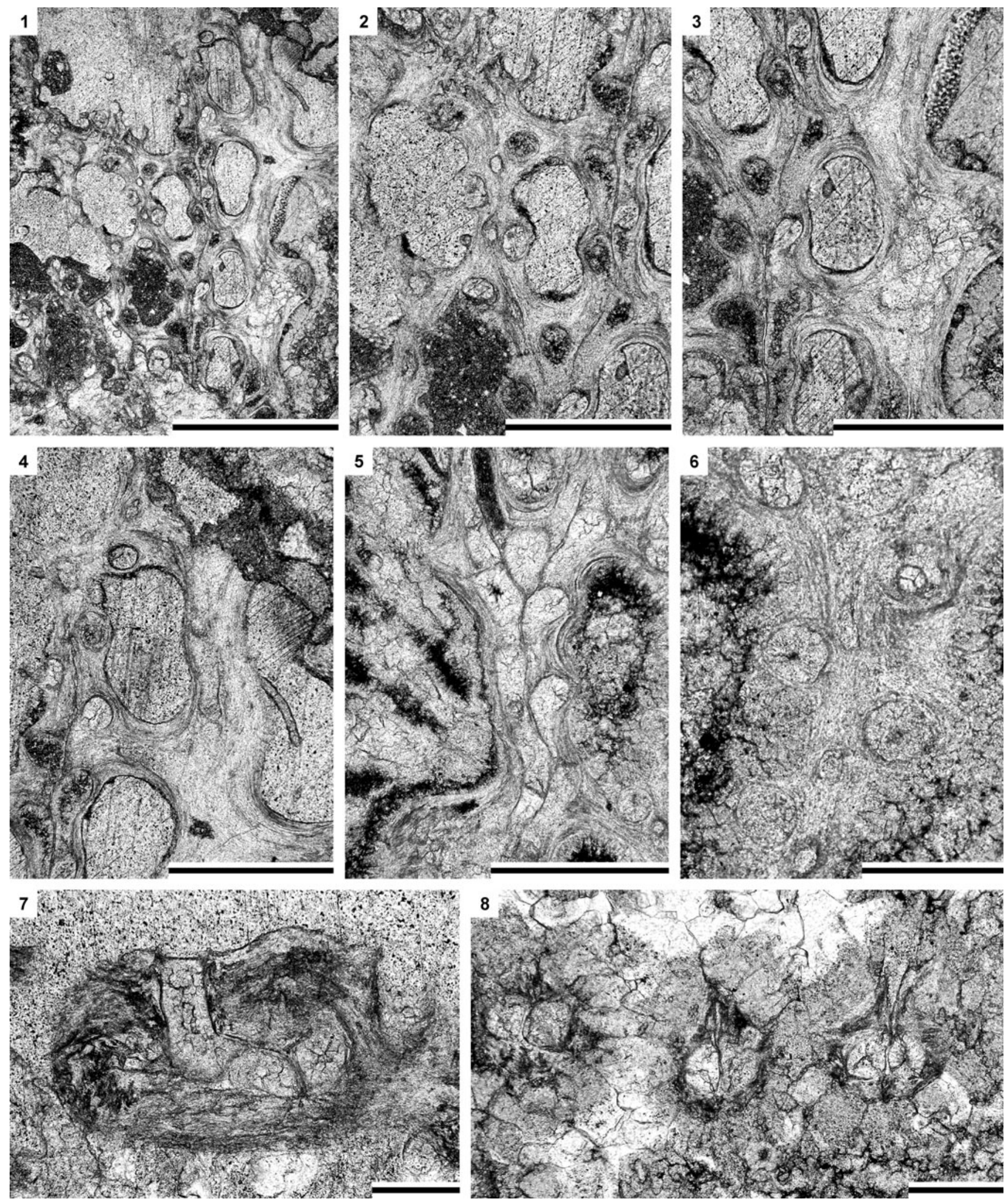

Figure 7. Moorephylloporina parvula $\mathrm{n}$. sp.: (1-4) tangential section showing autozooecial apertures and keel nodes, holotype, SMF 60668; (5) midtangentia section showing autozooecial chambers, paratype, SMF 60708; (6) tangential section showing autozooecial apertures and nodes, paratype, SMF 60708; (7) transverse section showing autozooecial chamber, paratype, SMF 60682; (8) transverse section showing autozooecial chambers, paratype, SMF 60702 . Scale bars $=1 \mathrm{~mm}(\mathbf{1})$ $0.5 \mathrm{~mm}(\mathbf{2}-\mathbf{5}) ; 0.2 \mathrm{~mm}(\mathbf{6}-\mathbf{8})$. 


\section{1}

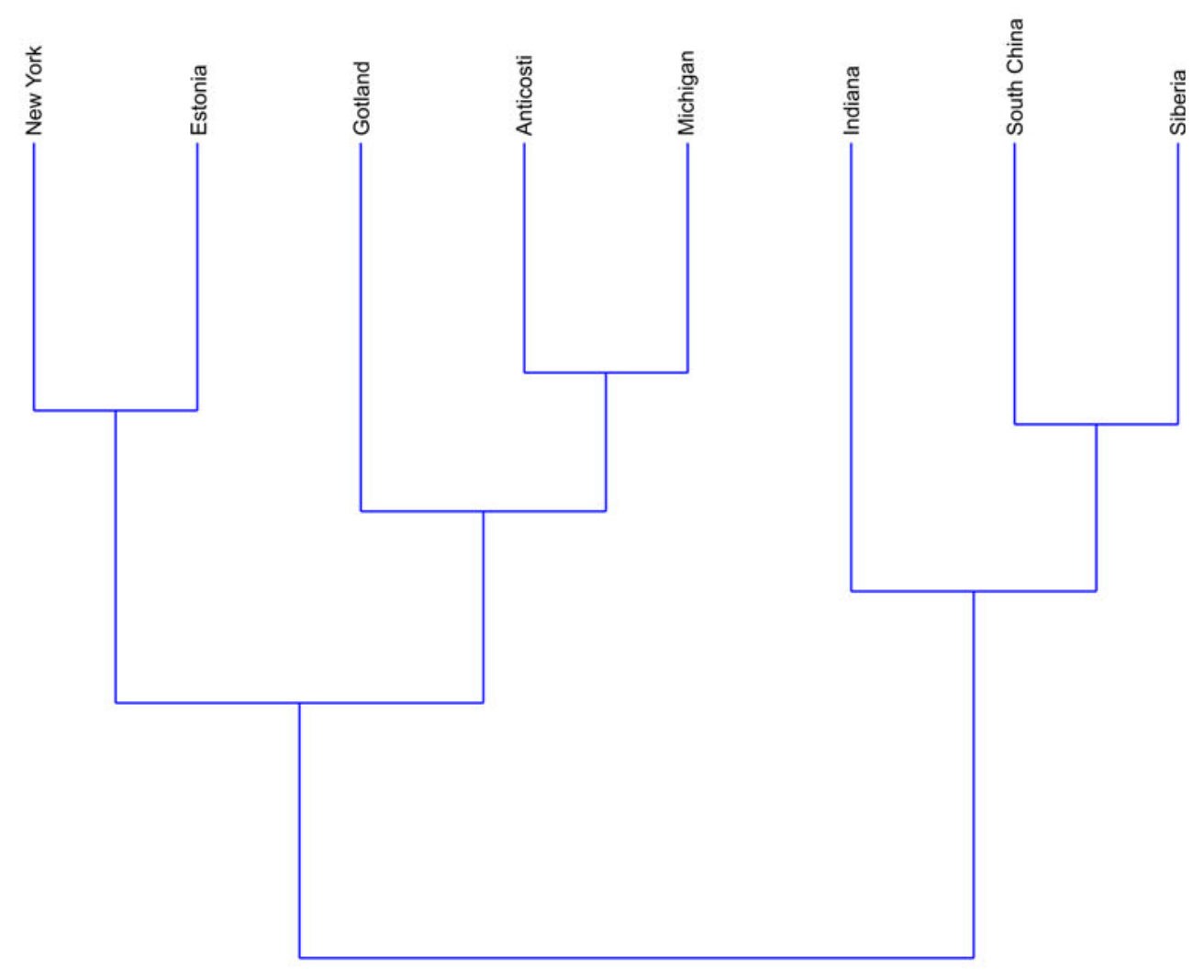

2

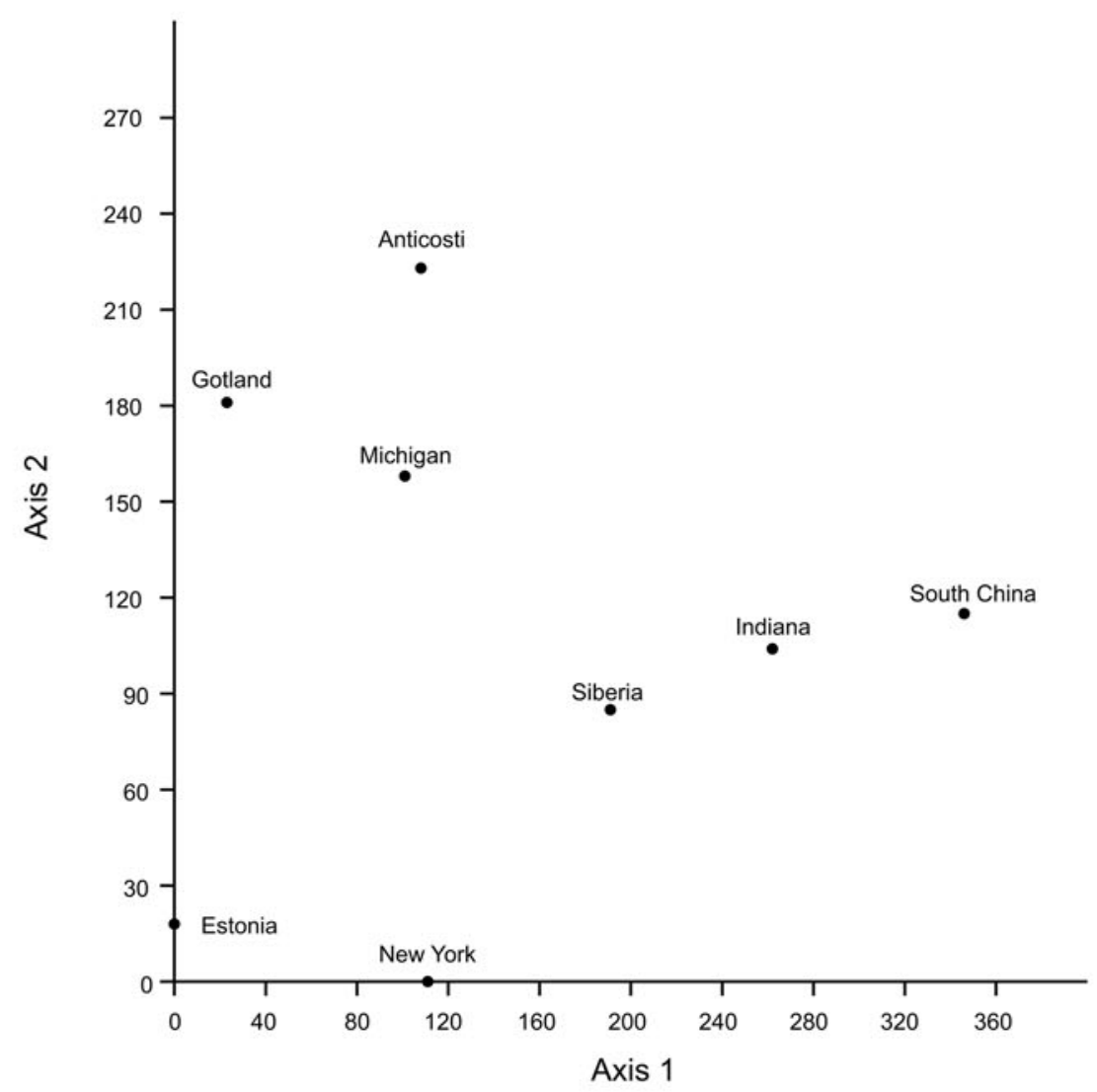


Figure 8. Paleobiogeographical affinities of Jiebei bryozoan association and selected Telychian bryozoan faunas (data from Anstey et al., 2003, updated 2020): (1) the hierarchical relationship based on pair-group cluster analysis (Euclidean similarity index); (2) detrended correspondence analysis. All analyses performed using PAST (Hammer et al., 2001).

Table 9. Distribution of bryozoan species in the Telychian localities of South China.

\begin{tabular}{|c|c|c|c|c|}
\hline \multirow{4}{*}{$\begin{array}{l}\text { Age } \\
\text { Locality } \\
\text { Formation } \\
\text { Reference }\end{array}$} & \multicolumn{3}{|c|}{ lower Telychian } & \multirow{4}{*}{$\begin{array}{c}\text { upper Telychian } \\
\text { Ningqiang, Shanxi } \\
\text { Ningqiang } \\
\text { Hu, 1982, } 1990\end{array}$} \\
\hline & Hanshan, Anhui & Guangyuan, Sichuan & Jiebei, Chongqing & \\
\hline & Chenxiacun & Cuijiagou & Hanchiatien & \\
\hline & Xia and Qi, 1989 & $\mathrm{Hu}, 1982$ & This paper & \\
\hline Asperopora sinensis n. sp. & & & $\mathrm{X}$ & \\
\hline Calloporella silurica $\mathrm{Hu}, 1982$ & & & & $\mathrm{x}$ \\
\hline Cyclotrypa solidoscens $\mathrm{Hu}, 1990$ & & & & $\mathrm{x}$ \\
\hline Cyphotrypa undulata $\mathrm{Hu}, 1990$ & & & & $\mathrm{x}$ \\
\hline Fistulipora ningqiangensis Hu, 1990 & & & & $\mathrm{x}$ \\
\hline Fistulipora guangyuanensis $\mathrm{Hu}, 1982$ & & $\mathrm{X}$ & $\mathrm{x}$ & \\
\hline Fistuliramus transversus $\mathrm{Hu}, 1982$ & & & & $\mathrm{X}$ \\
\hline Fistuliramus eximius $\mathrm{Hu}, 1982$ & & $\mathrm{x}$ & & \\
\hline Hallopora elegantula (Hall, 1852) & $\mathrm{X}$ & & & \\
\hline Hallopora raritabulata Xia and Qi, 1989 & $\mathrm{x}$ & & & \\
\hline Hallopora hanshanensis Xia and Qi, 1989 & $\mathrm{x}$ & & & \\
\hline Hallopora aggregata $\mathrm{Hu}, 1982$ & & & & $\mathrm{X}$ \\
\hline Hennigopora petaliformis $\mathrm{Hu}, 1982$ & & & & $\mathrm{x}$ \\
\hline Hennigopora multilamellosa $\mathrm{Hu}, 1982$ & & $\mathrm{X}$ & $\mathrm{X}$ & \\
\hline Hennigopora sp. indet. & & $\mathrm{x}$ & & \\
\hline Hennigopora sp. indet. & & & $\mathrm{X}$ & \\
\hline Homotrypa ningqiangensis $\mathrm{Hu}, 1990$ & & & & $\mathrm{X}$ \\
\hline Leioclema speciosa $\mathrm{Hu}, 1982$ & & & $\mathrm{X}$ & $\mathrm{x}$ \\
\hline Leioclema sp. indet. & & & & $\mathrm{x}$ \\
\hline Monotrypa shaanxiensis $\mathrm{Hu}, 1990$ & & & & $\mathrm{x}$ \\
\hline Orbignyella mui Yang, 1951 & & & & $\mathrm{x}$ \\
\hline Orbignyella curvata $\mathrm{Hu}, 1982$ & & & & $\mathrm{x}$ \\
\hline Orbignyella globata Yang, 1951 & & & & $\mathrm{x}$ \\
\hline Trematopora reflua Xia and Qi, 1989 & $\mathrm{x}$ & & & \\
\hline Trematopora sinensis $\mathrm{Hu}, 1982$ & & $\mathrm{X}$ & & \\
\hline Trematopora sp. indet. & & $\mathrm{x}$ & & \\
\hline Trematopora jiebeiensis $\mathrm{n}$. $\mathrm{sp}$. & & & $\mathrm{X}$ & \\
\hline Trematopora tenuis n. sp. & & & $\mathrm{x}$ & \\
\hline Moorephylloporina parvula $\mathrm{n} . \mathrm{sp}$. & & & $\mathrm{x}$ & \\
\hline
\end{tabular}

continuous, with obverse keel, nodes, peristome, and across dissepiments. Outer lamellar skeleton thin to moderately thick. Vesicular skeleton present.

Etymology.-The species name refers to the small size of this species (Latin parvulus = very small).

Remarks.-Moorephylloporina parvula n. sp. is similar to Moorephylloporina delicata (Nekhoroshev, 1961) from the Llandovery of Siberia. The new species differs in its wider branches (branch width $0.19-0.29 \mathrm{~mm}$ vs. 0.16-0.19 mm [Nekhoroshev, 1961], respectively) and smaller fenestrules (fenestrule width $0.16-0.26 \mathrm{~mm}$ vs. $0.30-0.40 \mathrm{~mm}$ [Nekhoroshev, 1961], respectively; fenestrule length 0.34-0.46 mm vs. 0.58-0.60 mm [Nekhoroshev, 1961], respectively).

\section{Discussion}

In the Jiebei section, bryozoans occur exclusively in the carbonate interbeds in the lower part of the Hanchiatien Formation (Figs. 2, 3). Characterized by reticular colonies, Moorephylloporina can be found in all types of facies in these meter-scale reefs, indicating its eurytopic nature. However, such distribution could be also explained by taphonomic processes and postmortem transportation of Moorephylloporina fragments from reef areas inhabited by this species within the whole reef. Although the abundance of Moorephylloporina is relatively low in the framestone (Fig. 3.3) of the reefs, these fenestellid bryozoans provide hard substrata for Fistulipora and Asperopora. In contrast, branched Trematopora and Leioclema tend to occur out of the reef core (framework) (Fig. 3.1). Like Champlainopora (Atactotoechus) chazyensis Ross, 1963 in some Ordovician reefs (e.g., Cuffey et al., 2002), Trematopora and Leioclema might have formed reef-flank thickets developed under more agitated conditions. Moorephylloporina, Trematopora, and Leioclema represent pioneering bryozoans that were able to grow on weakly lithified substrata. In contrast, encrusting Fistulipora, Hennigopora, and Asperopora relied more on hardgrounds and occupied a large proportion in the reefs, layered on top of one another and so forming densely compact framestones ( $\mathrm{Li}$ et al., 2018) that represent a typical crust-mound stage from an evolutionary ecological perspective (Cuffey, 2006).

The Telychian of the Upper Yangtze Platform is characterized by terrigenous sediments (e.g., Rong et al., 2012), and bryozoans are mostly reported from interbedded argillaceous limestones (or marls) (Hu, 1982, 1990; Xia and Qi, 1989), probably related to internal-wave deposits (Li et al., 2018) in some cases. Systematic studies of Telychian bryozoans are limited 
compared to other macrofossil groups, e.g., brachiopods, trilobites, and corals. Here, we provide the first detailed comparison of the composition of the bryozoan fauna (Table 9). In the South China block, Asperopora and Moorephylloporina are only reported from the Hanchiatien Formation, whereas Fistulipora and Trematopora are widely distributed across the platform during the early Telychian, recorded in all three studied sections in the literature. Apart from the Jiebei section, Hennigopora and Leioclema have been documented from one other Telychian section, indicating a moderately wide paleogeographic distribution. It is worth noting that bryozoans in some sections are not diverse, or else have been inadequately sampled. Further studies should be carried out to confirm their distributional patterns on the platform.

Bryozoans from the Jiebei section belong to genera with predominantly cosmopolitan distributions during the Telychian. Outside of South China, representatives of the genus Fistulipora are known from North America and Siberia. Species of Hennigopora are known from the USA (New York, Indiana) and Siberia. The genus Leioclema is known from North America, Europe (England, Ukraine), and Tuva (Russia). Records of Asperopora are known from North America (New York, Canada), Sweden (Gotland), and Siberia, whereas Trematopora is known from North America and China. The fenestrate genus Moorephylloporina is largely restricted to the Ordovician, except for two species from the lower Silurian (Llandovery) of Siberia, and the new species described here. It disappeared during the lower Silurian.

The early Silurian was a period of exceptional cosmopolitanism for benthic species with dispersive larvae (e.g., Cocks, 2001). Bryozoans, as in other groups of benthic organisms, showed low provinciality (e.g., Tuckey, 1990; Anstey et al., 2003; McCoy and Anstey, 2010; Buttler et al., 2013). The cluster analysis and detrended correspondence analysis (Fig. 8) reveal distinct clustering of South China with Siberia and Indiana (USA). Bryozoan faunas of Estonia seem to be close to those of New York (USA), whereas Gotland (Sweden) clusters with Anticosti (Canada) and Michigan (USA). These results agree with existing paleogeographical reconstructions for the early Silurian (e.g., Cocks and Torsvik, 2002). However, most existing references for bryozoans in the lower Silurian need critical reassessment, because the descriptions are often far from present-day standards.

\section{Acknowledgments}

This study was supported by the Youth Innovation Promotion Association of the Chinese Academy of Sciences (CAS) no. 2019310, the Natural Science Foundation of China (NSFC) no. 41702003 , and CAS no. XDB26000000 and XDA19050101. We are grateful to B. Leipner-Mata (GeoZentrum Nordbayern, Erlangen, Germany) for the preparation of thin sections and to S. Yu (Nanjing Institute of Geology and Palaeontology) for assistance in the field. This study is a contribution to the International Geoscience Projects (IGCP) 653 and 668. QJL is thankful for his invitation as a guest researcher at GeoZentrum Nordbayern. P.D. Taylor (London) and H.A. Nakrem (Oslo) are thanked for their helpful and constructive reviews of the manuscript.

\section{Supplementary material}

To view supplementary material for this article, please visit https://doi.org/10.1017/jpa.2020.86

\section{References}

Anstey, R.L., Pachut, J.F., and Tuckey, M.E., 2003, Patterns of bryozoan endemism through the Ordovician-Silurian transition: Paleobiology, v. 29, p. 305-328. doi:10.1666/0094-8373(2003)029<0305:POBETT>2.0.CO;2.

Astrova, G.G., 1959, Silurian bryozoans of the central and western Tuva: Trudy Paleontologicheskogo Instituta Akademii Nauk SSSR, v. 79, p. 1-74. [in Russian]

Astrova, G.G., 1960, Silurian fistuliporids from northern territories of USSR: Trudy Komy Filiala Akademii Nauk SSSR, Syktyvkar, v. 1, 352-376. [in Russian]

Astrova, G.G., 1964, A new order of the Paleozoic Bryozoa: Paleontologicheskii Zhurnal, no. 2, p. 22-31. [in Russian]

Astrova, G.G., 1965, Morphology, history of development and system of the Ordovician and Silurian Bryozoa: Trudy Paleontologicheskogo Instituta Akademii Nauk SSSR, v. 106, p. 1-432. [in Russian]

Astrova, G.G., and Morozova, I.P., 1956, On the systematics of the bryozoans of the order Cryptostomata: Doklady Akademii Nauk SSSR, v. 110, p. 661664. [in Russian]

Baily, W.H., 1871, Palaeontological remarks in Traill, W.A. and Egan, F.W. (eds.), Explanatory Memoir to Accompany Sheets 49, 50 and Part of 61 of the Maps of the Geological Survey of Ireland Including the Country Around Downpatrick, and the Shores of Dundrum Bay and Strangford Lough, County of Down: Dublin, Ireland, Alexander Thom, p. 22-23.

Bassler, R.S., 1906, The bryozoan fauna of the Rochester Shale: United States Geological Survey Bulletin, v. 292, p. 1-137.

Bassler, R.S., 1911a, Corynotrypa, a new genus of tubuliporoid Bryozoa: Proceedings of the United States National Museum, v. 39, 497-527.

Bassler, R.S., 1911b, The early Paleozoic Bryozoa of the Baltic Provinces: Bulletin of the Smithsonian Institution, United States National Museum, v. 77, p. $1-382$.

Bassler, R. S., 1936, Nomenclatorial notes on fossil and Recent Bryozoa: Journal of the Washington Academy of Science, v. 26, p. 156-162.

Bassler, R.S., 1952, Taxonomic notes on genera of fossil and Recent Bryozoa: Journal of the Washington Academy of Sciences, v. 42, p. 381-385.

Borg, F., 1926, Studies on Recent cyclostomatous Bryozoa: Zoologiska Bidrag från Uppsala, v. 10, p. 181-507.

Buttler, C.J., Wyse Jackson, P.N., Ernst, A., and McKinney, F.K., 2013, A review of the early Palaeozoic biogeography of bryozoans, in Harper, D., and Servais, T., eds., Early Palaeozoic Palaeobiogeography and Palaeogeography: Geological Society of London, Memoirs, v. 38, p. 145-155, doi:10.1144/M38.12.

Chen, X., 1986, On Streptograptus and its paleoautecology, in Palaeontological Society of China, ed., Selected Papers from the 13th and 14th Annual Conventions of the Palaeontological Society of China: Hefei, China, Anhui Science and Technology Publishing House, p. 115-137. [in Chinese]

Cocks, L.R.M., 2001, Ordovician and Silurian global geography: Journal of the Geological Society, v. 158, p. 197-210.

Cocks, L.R.M., and Torsvik, T.H., 2002, Earth geography from 500 to 400 million years ago: A faunal and palaeomagnetic review: Journal of the Geological Society, London, v. 159, p. 631-644. doi:10.1144/0016-764901-118.

Cuffey, R.J., 2006, Bryozoan-built reef mounds-The overview from integrating recentstudies with previous investigation: Courier Forschungsinstitut Senckenberg, v. 257, p. 35-48.

Cuffey, R.J., Robison, M.R., and Mehrtens, C.J., 2002, Garden Island-The earliest (and first-illustrated) bryozoan reef in North America (basal Chazyan, early Middle Ordovician; Lake Champlain, New York-Vermont): Geological Society of America, Abstracts with Program, v. 34, p. A-72.

Deng, X.J., Wang, G., and Li, Y., 2012, Sedimentary characteristics at the top of the Shihniulan Formation (late Aeronian, Silurian) and their implications for identification of the shore line in Tongzi, northern Guizhou: Journal of Stratigraphy, v. 36, p. 718-722 [in Chinese].

Duncan, H., 1939, Trepostomatous Bryozoa from the Traverse Group of Michigan: Contributions from the Museum of Paleontology, University of Michigan, v. 5, 171-270.

Dzik, J., 1981, Evolutionary relationships of the early Paleozoic cyclostomatous Bryozoa: Palaeontology, v. 24, p. 827-862.

Ehrenberg, C.G., 1831, Symbolae Physicae, seu Icones et Descptiones Corporum Naturalium Novorum aut Minus Cognitorum, quae ex Itineribus per Libyam, Aegiptum, Nubiam, Dongalaam, Syriam, Arabiam et Habessiniam, Studia Annis 1820-25, Redirent, Pars Zoologica, 4, Animalia Evertebrata exclusis Insectis: Berlin, 10 pls. 
Eichwald, E., 1855, Beitrag zur geographischen Verbreitung der fossilen Tiere Russlands: Bulletin de la Société des Naturelles de Moscovian, v. 28, p. $433-466$.

Elias, M.K., and Condra, G.E., 1957, Fenestella from the Permian of West Texas: Memoirs of the Geological Society of America, v. 70, p. 1-158.

Ernst, A., Munnecke, A., and Oswald, I., 2015, Exceptional bryozoan assemblage of a microbial-dominated reef from the early Wenlock of Gotland, Sweden. GFF, v. 137, p. 102-125. doi:10.1080/11035897.2014.997543.

Ernst, A., Brett, C.E., and Wilson, M.A., 2019, Bryozoan fauna from the Reynales Formation (lower Silurian, Aeronian) of New York, USA: Journal of Paleontology, v. 93, p. 628-657. doi:10.1017/jpa.2018.101.

Foerste, A.F., 1887, The Clinton Group of Ohio, Part 3: Bulletin of the Scientific Laboratories of Denison University, v. 2, 149-176.

Foerste, A.F., 1895, Fossils of the Clinton Group of Ohio and Indiana: Report of the Geological Survey of Ohio, v. 7, p. 516-601.

Geng, L.Y., 1986, Lower Silurian chitinozoans from Bayu of Daozhen, Guizhou and Dazhongba of Yichang, Hubei: Acta Palaeontologica Sinica v. 25, p. 117-128. [in Chinese with English summary]

Geng, L.Y, Qian, Z.S., Ding, L.S., Wang, Y., Wang, G.X., and Cai, X.Y., 1997, Silurian chitinozoans from the Yangtze Region: Palaeoworld, v. 8, p. 1-152

Hall, J., 1847, Palaeontology of New York. Volume 1, Containing Descriptions of the Organic Remains of the Lower Division of the New-York System: Albany, New York, D. Appleton \& Company, 337 p.

Hall, J., 1851, New genera of fossil corals from the report of James Hall, On the Palaeontology of New York: American Journal of Science and Arts, v. 11, p. 398-401.

Hall, J., 1852, Palaeontology of New York, Volume 2, Organic Remains of the Lower Middle Division of the New York System: New York Geological Survey, Natural History of New York, v. 6, p. 40-52, 144-173.

Hall, J., 1858, Paleontology of Iowa, in Hall, J., and Whitney, J.D., Report of the Geological Survey of the State of Iowa, Embracing the Results of Investigations Made During Portions of the Years 1855, 56 \& 57: Palaeontology, Volume 1, Part 2: Des Moines, Legislature of Iowa, p. 473-724.

Hall, J., 1885, On the mode of growth and relations of the Fenestellidae: Report of the State Geologist of New York for the Year 1884, p. 35-45.

Hall, J., 1886, Bryozoa of the Upper Helderberg Group; plates and explanations: State Geologist of New York, Annual Report for 1885, p. 25-53.

Hammer, Ø., Harper, D.A.T., and Ryan, P.D., 2001, PAST: Paleontological statistics software package for education and data analysis: Palaeontologia Electronica, v. 4, p. 1-9.

Hu, Z.X., 1982, Silurian Bryozoa from northern Sichuan and southern Shaanxi: Acta Palaeontologica Sinica, v. 21, p. 290-301. [in Chinese]

Hu, Z.X., 1990, Telychian (Silurian) Bryozoa from Ningqiang, S. Shaanxi: Acta Palaeontologica Sinica, v. 29, p. 600-611. [in Chinese]

King, W., 1849, On some families and genera of corals: Annals and Magazine of Natural History, v. 2, p. 388-390.

Kopajevich, G.V., 1975, Silurian Bryozoa of Estonia and Podolia (Cryptostomata and Rhabdomesonata): Trudy Paleontologischeskogo Instituta Akademiya Nauk SSSR, v. 151, p. 5-153. [in Russian]

Kopajevitch, G.V., 1984, Atlas of Bryozoa from the Ordovician, Silurian and Devonian of Mongolia: Trudy Sovmestnoi Sovetsko-Mongolsoi Paleontologischeskoi Ekspeditsii, v. 22, p. 1-164. [in Russian]

Li, Q.J., Wang, Y.Y., Li, Y., Ma, J.Y., Zhang, Y.Y., Deng, X.J., and Cai, X.Y., 2012, Muddy sediments constrain reef-bank growth in the Hanjiadian Formation, Tongzi, northern Guizhou, SW China: Acta Palaeontologica Sinica, v. 51, p. 127-136. [in Chinese]

Li, Q.J., Ernst, A., Munnecke, A., Yu, S., Li, Y., and Deng, X.J., 2018, Early Silurian (Telychian) bryozoan reefs in the epeiric sea of South China: Are heterotroph metazoan buildups promoted by internal waves?: Sedimentary Geology, v. 376, p. 50-59. doi:10.1016/j.sedgeo.2018.07.008.

Lonsdale, W., 1839, Corals, in Murchison, R.I., ed., The Silurian System, Part 2, Organic Remains: London, John Murray, p. 675-694.

Ma, J.-Y., Buttler C.J., and Taylor, P.D., 2014, Cladistic analysis of the 'trepostome' suborder Esthonioporina and the systematics of Palaeozoic bryozoans, in Rosso, A., Wyse Jackson, P.N., and Porter, J.S., eds., Bryozoan Studies 2013: Studi Trentini di Scienze Naturali, v. 94 p. $153-161$.

McCoy, V.E., and Anstey, R.L., 2010, Biogeographic associations of Silurian bryozoan genera in North America, Baltica and Siberia: Palaeogeography, Palaeoclimatology, Palaeoecology, v. 297, p. 420-427. doi:10.1016/j. palaeo.2010.08.025.

M'Coy, F., 1849, On some new genera and species of Palaeozoic corals and Foraminifera: Annals and Magazine of Natural History, v. 2, p. 119-136.

Miller, S.A., 1889, North American Geology and Paleontology for the Use of Amateurs, Students and Scientists: Cincinnati, Ohio, Western Methodist Book Concern, $664 \mathrm{p}$.

Miller, T.G., 1962, Some Wenlockian fenestrate Bryozoa: Palaeontology, v. 5, p. $540-549$

Mu, E.Z., 1962, Silurian System of China: Beijing, Science Press, 95 p. [in Chinese]
Nekhoroshev, V.P., 1956, Class Bryozoa: Trudy VSEGEI, n. ser., v. 12, p. 42 49. [in Russian]

Nekhoroshev, V.P., 1961, Ordovician and Silurian bryozoans of the Siberian Plate: Trudy VSEGEI, new ser., v. 41, p. 1-246. [in Russian]

Nicholson, H.A., 1879, On the Structure and Affinities of the 'Tabulate Corals' of the Paleozoic Period, with Critical Descriptions of Illustrative Species: Edinburgh, William Blackwood and Sons, 342 p.

Orbigny, A. d', 1849, Description des quelques genres nouveaux de mollusques bryozoaires: Revue et Magazine de Zoologie Pure et Appliquée, v. (2) 1, p. 499-504.

Owen, D.E., 1965, Silurian Polyzoa from Benthall Edge, Shropshire: Bulletin of the British Museum (Natural History), Geology, v. 10, p. 95-117.

Owen, D.E., 1969, Wenlockian Bryozoa from Dudley, Niagara, and Gotland and their palaeogeographic implications: Palaeontology, v. 12, p. 621-636.

Perry, T.G., and Hattin, D.E., 1960, Osgood (Niagaran) bryozoans from the type area [Indiana]: Journal of Paleontology, v. 34, p. 695-710.

Prout, H. A., 1859, Third series of descriptions of Bryozoa from the Palaeozoic rocks of the western states and territories: Transactions of the St. Louis Academy of Science, v. 1, p. 443-452.

Pushkin, V.I., 1976, New species of Ordovician and Silurian Bryozoa of the Brest Depression, in Akimets, V.S., and Govetskii, G.I., eds., New Species of the Fossil Plants and Animals of Belarus: Minsk, Belarus, Nauka i Tekhnika, p. 3-40. [in Russian]

Pushkin, V. I., 1977, New genus of Ordovician bryozoan: Paleontologicheskii Zhurnal, 1977, no. 4, p. 67-72. [in Russian]

Rong, J.Y., and Cocks, L.R.M., 2014, Global diversity and endemism in early Silurian (Aeronian) brachiopods: Lethaia, v. 47, p. 77-106. doi:10.1111/ let. 12042.

Rong, J.Y., Chen, X., Wang, C.Y., Geng, L.Y., Wu, H.J., Deng, Z.Q., Chen, T.E., and Xu, J.T., 1990, Some problems concerning the correlation of the Silurian rocks in South China: Journal of Stratigraphy, v. 14, p. 161177. [in Chinese]

Rong, J.Y., Chen, X., Su, Y.Z., Ni, Y.N., Zhan, R.B., Chen, T.E., Fu, L.P., Li, R.Y., and Fan, J.X., 2003, Silurian paleogeography of China: in Landing, E., and Johnson, M.E., eds., Silurian Lands and Seas: Paleogeography Outside of Laurentia: New York, New York State Museum, p. 243-298.

Rong J.Y., Wang Y., and Zhang X.L., 2012, Tracking shallow marine red beds through geological time as exemplified by the lower Telychian (Silurian) in the Upper Yangtze Region, South China: Science China Earth Sciences, v. 55, p. 699-713. doi:10.1007/s11430-012-4376-5.

Ross, J.R.P., 1963, Chazyan (Ordovician) leptotrypellid and atactotoechid Bryozoa: Palaeontology, v. 5, p. 727-739.

Shi, G.R., 1993, Multivariate data analysis in paleoecology and paleobiogeography-A review: Palaeogeography, Palaeoclimatology, Palaeoecology, v. 105 , p. 199-234.

Shrubsole, G.W., and Vine, G.R., 1882, The Silurian species of Glauconome and a suggested classification of the Palaeozoic Polyzoa: Geological Magazine, v. 9, p. 381-382.

Siegfried, P., 1963, Bryozoen in Steinkernerhaltung aus ordovizischen Geschieben: Paläontologische Zeitschrift, v. 37, 135-146.

Silliman, B., Silliman, B., Jr., and Dana, J.D., 1851, New genera of fossil corals from the report of James Hall, On the Palaeontology of New York: American Journal of Sciences and Arts, v. 2, p. 398-401.

Southwest Institute of Geologic Sciences, 1978, Palaeontological Atlas of Southwest China, Sichuan Volume (1) Sinian-Devonian: Beijing, Geological Publishing House, 617 p. [in Chinese]

Suttner, T., and Ernst, A., 2007, Upper Ordovician Bryozoa of the Pin Formation (Spiti Valley, northern India): Palaeontology, v. 50, p. 1485-1518. doi:10.1111/j.1475-4983.2007.00726.x.

Ting W.J., 1930, The Fengninian Series in Kueichow: Bulletin of the Geological Society of China, v. 9, p. 95-96.

Tuckey, M.E., 1990, Distributions and extinctions of Silurian Bryozoa, in McKerrow, W.S., and Scotese, C.R., eds., Palaeozoic Palaeogeography and Biogeography: Geological Society of London, Memoirs, v. 12, p. 197-206.

Ulrich, E.O., 1882, American Paleozoic Bryozoa: The Journal of the Cincinnati Society of Natural History, v. 5, p. 121-175, 233-257.

Ulrich, E.O., 1883, American Palaeozoic Bryozoa: The Journal of the Cincinnati Society of Natural History, v. 6, p. 245-279.

Ulrich, E.O., 1884, American Palaeozoic Bryozoa: The Journal of the Cincinnati Society of Natural History, v. 8, p. 24-51.

Ulrich, E.O., 1888, A list of the Bryozoa of the Waverly Group in Ohio; with descriptions of new species: Denison University, Bulletin of Scientific Laboratories, v 4, p. 62-96.

Ulrich, E.O., 1890, Palaeozoic Bryozoa, III: Report of the Geological Survey of Illinois, v. 8, p. 283-688.

Ulrich, E.O., 1893, On lower Silurian Bryozoa of Minnesota: The Geologica and Natural History Survey of Minnesota, Final Report, v. 3, p. 96-332. 
Ulrich, E.O., 1896, Bryozoa, in Eastman, C., ed., Zittel's Textbook of Palaeontology, Volume 1: London, Macmillan, p. 25-291.

Ulrich, E.O., and Bassler, R.S., 1904, A revision of Palaeozoic Bryozoa: Smithsonian Miscellaneous Collections, v. 45, p. 256-294.

Utgaard, J., 1983, Systematic descriptions for the order Cystoporata, in Robison R.A., ed., Treatise on Invertebrate Paleontology, Part G, Bryozoa 1 (revised): Boulder, Colorado, and Lawrence, Kansas, Geological Society of America (and University of Kansas Press), p. G357-G439.

Vinassa de Regny, P. E., 1915, Fossili Ordoviciani del Capolago (Seekopf) presso il Passo di Volaia (Alpi Carniche): Palaeontographia Italica, v. 21, p. $97-115$.

Xia, F.S., and Qi, D.L., 1989, Trepostomatous bryozoans from the Chenxiacun Formation (late early Silurian) of Hanshan, Anhui: Acta Micropalaeontologica Sinica, v. 6, p. 75-90. [in Chinese]
Yang, J., 1951, Two new species of Bryozoa from the middle Silurian of Kuanguan, Szechuan: Bulletin of the Geological Society of China, v. 31, p. $85-88$.

Yang J., and Xia, F.-S., 1974, Bryozoans, in A Handbook of the Stratigraphy and Palaeontology of Southwest China: Beijing, Science Press, p. 195, 240, 275, 308. [in Chinese]

Yin, T.H., 1949, Subdivision and correlation of Silurian rocks in South China: Bulletin of the Geological Society of China, v. 29, p. 1-61. [in Chinese]

Zhan, R.B., and Jin, J.S., 2007, Ordovician-Early Silurian (Llandovery) Stratigraphy and Palaeontology of the Upper Yangtze Platform, South China: Beijing, Science Press, 169 p.

Accepted: 9 September 2020 